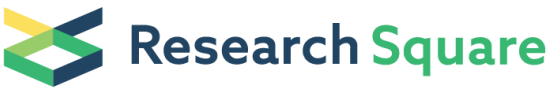 \\ Preprints are preliminary reports that have not undergone peer review. \\ They should not be considered conclusive, used to inform clinical practice, \\ or referenced by the media as validated information.
}

\section{Long-term culture expanded alveolar macrophages restore full epigenetic identity in vivo}

\section{Sethuraman Subramanian}

Aix Marseille University, CNRS, INSERM, CIML, Marseille, France https://orcid.org/0000-0002-4917084X

\section{Clara Busch}

Center for Regenerative Therapies Dresden (CRTD), Technische Universität Dresden, Dresden, Germany

\section{Kaaweh Molawi}

Max-Delbrück-Centrum für Molekulare Medizin in der Helmholtzgemeinschaft (MDC), Berlin, Germany

\section{Laufey Geirsdottir}

Aix Marseille University, CNRS, INSERM, CIML, Marseille, France

Julien Maurizio

Innovarion, Paris, France

\section{Stephanie Vargas-Aguilar}

Center for Regenerative Therapies Dresden (CRTD), Technische Universität Dresden, Dresden, Germany Hassiba Belahbib

Aix Marseille University, CNRS, INSERM, CIML, Marseille, France

\section{Gregory Gimenez}

Marseille University, CNRS, INSERM, CIML, Marseille, France

\section{Bérengère de Laval}

Centre d'Immunologie de Marseille - Luminy, Aix Marseille University, Centre National de la Recherche

Scientifique, Institut National de la Santé et de la Recherche Médicale https://orcid.org/0000-0002-56162338

\section{Ridzky Yuda}

Center for Regenerative Therapies Dresden (CRTD), Technische Universität Dresden, Dresden, Germany

\section{Michaela Burkon}

Center for Regenerative Therapies Dresden (CRTD), Technische Universität Dresden, Dresden, Germany

\section{Sara Gholamhosseinian Najjar}

Center for Regenerative Therapies Dresden (CRTD), Technische Universität Dresden, Dresden, Germany https://orcid.org/0000-0001-8457-2564

\section{Prashanth Kumar Kandalla}

Center for Regenerative Therapies Dresden (CRTD), Technische Universität Dresden, Dresden, Germany

\section{Sandrine Sarrazin}

Aix Marseille Univ, CNRS, INSERM, CIML

\section{Lena Alexopoulou}


Centre d'Immunologie de Marseille https://orcid.org/0000-0003-4619-697X

Jérémy Favret

Center for Regenerative Therapies Dresden (CRTD), Technische Universität Dresden, Dresden, Germany

Michael Sieweke ( $\nabla$ sieweke@ciml.univ-mrs.fr)

Centre d'Immunologie de Marseille - Luminy, Aix Marseille University, Centre National de la Recherche Scientifique, Institut National de la Santé et de la Recherche Médicale/Max-Delbr https://orcid.org/00000002-3228-9537

\section{Technical Report}

\section{Keywords:}

Posted Date: December 22nd, 2021

DOl: https://doi.org/10.21203/rs.3.rs-756350/v1

License: (c) (1) This work is licensed under a Creative Commons Attribution 4.0 International License. Read Full License

Version of Record: A version of this preprint was published at Nature Immunology on February 24th, 2022. See the published version at https://doi.org/10.1038/s41590-022-01146-w. 
Long term culture expanded alveolar macrophages restore full epigenetic identity in vivo

Sethuraman Subramanian ${ }^{1,2,3, \#}$, Clara Jana-Lui Busch ${ }^{1, \#}$, Kaaweh Molawi ${ }^{2,3}$, Laufey Geirsdottir², Julien Maurizio ${ }^{4}$, Stephanie Vargas-Aguilar ${ }^{1,2,3}$, Hassiba Belahbib², Gregory Gimenez ${ }^{2}$, Bérengère de Laval ${ }^{2}$, Ridzky Anis Advent Yuda ${ }^{1}$, Michaela Burkon ${ }^{1}$, Sara Gholamhosseinian Najjar $^{1}$, Prashanth Kumar Kandalla ${ }^{1}$, Sandrine Sarrazin ${ }^{1,2}$, Lena Alexopoulou² and Michael H. Sieweke ${ }^{1,2}$

1 Center for Regenerative Therapies Dresden (CRTD), Technische Universität Dresden, Dresden, Germany,

2 Aix Marseille University, CNRS, INSERM, CIML, Marseille, France

3 Max-Delbrück-Centrum für Molekulare Medizin in der Helmholtzgemeinschaft (MDC), Berlin, Germany

4 Innovarion, Paris, France

\# joint first author

\section{Abstract}

Alveolar macrophages (AM) are tissue resident macrophages of the lung that can be expanded in culture, but it is unknown to what extent culture affects their in vivo identity. Here we show that long term ex vivo expanded AM (exAM) maintain core AM gene expression but show culture adaptations related to adhesion, metabolism and proliferation. Strikingly, even after several months in culture exAM reacquired full transcriptional and epigenetic identity upon transplantation into the lung and could self-maintain in the natural niche long term. Changes in open chromatin regions (OCR) observed in culture were fully reversible in transplanted exAM (texAM) and resulted in a gene expression profile indistinguishable from resident AM. Our results demonstrate that long term proliferation of AM in culture does not compromise cellular identity in vivo. The demonstrated robustness of exAM identity provides new opportunities for mechanistic analysis and highlights the therapeutic potential of ex vivo expanded macrophages. 
47 - Specific tissue resident macrophage identity can be maintained through long term culture 48

49 - Long term proliferation does not compromise differentiated macrophage identity

- Epigenetic and transcriptional culture adaptations are fully reversible in vivo

53 - Rare example of long term cultured somatic cells restoring full epigenetic identity in natural $54 \quad$ niche in vivo

- Shuttling cells between ex vivo culture and natural niche in vivo provides a valuable system for genetic and biochemical investigation

59 - Robustness of macrophage identity through culture highlights potential of macrophage 60 based cellular therapies, including in respiratory disease such as COVID19 
Resident macrophages can be found in every tissue of the body where they fulfill diverse functions in homeostasis, immunity and repair $\underline{1}$. Organ specific functions and challenges induce functional specialization of macrophages resulting in highly diverse phenotypes of tissue macrophage populations $\underline{3} \underline{4}$.

The potential determinants and stability of macrophage identity have been a subject of intense investigation and debate $\underline{5} \underline{6}$. For example, in the lung, various transplantation protocols have shown adaptations of macrophages from different origin to the alveolar macrophage niche $\underline{4} \underline{7} \underline{8}$, which has led to the suggestion that the niche has a powerful, possibly dominant role ${ }^{5}$. Conversely, macrophages loose part of their tissue specific gene expression signature when placed in culture $\underline{1} \underline{10} \underline{11} \underline{12}$. This is likely due to the loss of critical environmental signals from the niche in vivo, as well as diverse new chemical and physical stimuli encountered in culture. It has remained unclear, however, whether these adaptations to the cell culture environment are permanent or reversible. In particular it is unknown, whether they involve irreversible changes in chromatin accessibility, or whether core epigenetic identity and the sensitivity to respond to correct environmental cues can be maintained through long periods and multiple rounds of cell division in culture.

Tissue culture models have been tremendously important for the advance of immunology but there are only few primary macrophage culture systems available. The most commonly used protocol is based on the differentiation of bone marrow cells in M-CSF/CSF-1 containing medium. Despite the appeal of being simple and accessible the protocol has major drawbacks. Since it involves differentiation from heterogeneous progenitor cells, it bears the problem of giving rise to a non-uniform population of cells of non-synchronized differentiation states. The inherent danger of this is exemplified by a similar differentiation protocol of bone marrow cells in GM-CSF, which turned out to contain a mixed population of macrophages and dendritic cells that went undetected in many previous studies $\underline{13}$. Thioglycolate-elicited peritoneal macrophages, another popular culture model, do not reflect a homeostatic cell population and contain an eosinophilic contamination $\underline{\underline{14}}$. Macrophage differentiation from monocytes, in particular from human donors, suffers from batch to batch variability. Cell culture has been critical for the generation of large numbers of immune cells, in particular T-cells, for cancer cellular therapies, but in contrast to T-cells, the proliferative capacity of macrophages in culture is very limited $\underline{15} \underline{16}$. All common macrophage culture protocols therefore have the drawback to only generate a limited number of cells and to be usable only short-term.

We have shown previously that alveolar macrophages constitutively express low levels of the anti-proliferative transcription factors MafB and cMaf, which enables them to access a network of self-renewal genes that are repressed by MafB and cMaf in other macrophage populations $\underline{17} \underline{18}$. As a consequence, alveolar macrophages are unique among tissue resident 
macrophages in that they can expand in culture to large numbers and proliferate for extended periods of time $\underline{18} \underline{19}$. It is therefore of high interest for experimental science and potential cellular therapy applications, whether macrophage identity can be maintained through long term proliferation in culture.

Here we have addressed these questions by comparing transcriptional and epigenetic identity of alveolar macrophages in long term culture before and after re-transplantation into the natural niche environment in the lung. We observed that adaptations of alveolar macrophages to the culture environment were transient and did not compromise functional long-term integration into the natural alveolar niche of the lung. Even after long term proliferation in culture, ex vivo expanded alveolar macrophages showed a transcriptional and epigenetic signature after transplantation that was indistinguishable from resident AM that had never transitioned through culture. This indicated that alveolar macrophages sustained sensitivity to critical environmental cues encountered in vivo through long periods of culture. Together our findings establish a unique macrophage culture system of high fidelity for shuttling between ex vivo experimental manipulation and in vivo validation. It also demonstrates that macrophage expansion ex vivo can provide large scale preparations for cellular therapy applications with maintained normal macrophage identity in vivo.

\section{Results}

\section{Alveolar macrophages show a massive expansion potential in long term culture}

We have shown previously that alveolar macrophages obtained from broncho-alveolar lavage (BAL) can be cultured $\underline{19}$ and expanded in GM-CSF containing medium $\underline{18}$. Here we have further characterized such cultures and named them "exAM" for expanded AM (Fig.1A). We observed that these cells could be kept in continuous culture for at least 10 months, resulting in 33 theoretical population doublings and an amplification factor of $10^{10}$ (Fig.1B). This correlated with a three- to four-fold higher percentage of cells in S-phase of the cell cycle in exAM compared to fresh BAL (Fig.1C). Furthermore, the exAM cultures could also be taken through freeze/thaw cycles without compromising growth capacity (not shown). Together these observations indicated an enormous, potentially unlimited expansion potential of exAM.

\section{Expanded alveolar macrophages maintain characteristic phenotype and function in long} term culture

We further analyzed whether exAM cultures maintained typical macrophage phenotypic and functional characteristics in culture. We observed that long term exAM cultures had a typical AM phenotypic appearance and Diff-Quik dye staining properties (Fig.2A). Furthermore, the exAM cultures showed macrophage typical acidified lysosomal structures as indicated by acridine orange staining. They also showed labeling with Magic Red dye, a sensor of enzymatic 
145 activity of the lysosomal protease Cathepsin B (Fig.2B). The cells could also take up 146 fluorescently labelled latex beads, indicating that they are capable of phagocytosis (Fig.2C). In order to quantify this and demonstrate active phagocytosis, we took advantage of zymosan labelled with the $\mathrm{pH}$ sensitive dye pHRhodo that only fluoresces upon acidification of the phagocytosed particles in the lysosomes. FACS quantification of this dye showed the same strong signal in exAM cultures as in freshly harvested $\mathrm{BAL}$, that was present only at $37^{\circ} \mathrm{C}$ but not at $4^{\circ} \mathrm{C}$ (Fig.2D). This indicated that exAM maintain full ability of AM to phagocytose pathogen associated material in an active metabolic process that involves the uptake into acidic and enzymatically active lysosomal structures. In order to analyze whether exAM could also mount a macrophage typical immune response, we stimulated exAM cultures with E.coli LPS as a TLR4 stimulating PAMP and mimetic of infection with a gram-negative bacterial pathogen. As shown in Fig.2E, LPS stimulation resulted in production of reactive oxygen species (ROS) in exAM cultures similar to fresh BAL. Together our results indicated that exAM cultures showed typical macrophage functional characteristics.

We further analyzed whether exAM not only maintained general macrophage functions but also a specific AM identity in long term culture. Flow cytometric analysis demonstrated that exAM maintained typical macrophage surface marker staining including the characteristic AM markers SiglecF and CD11C (Fig.2F), which were also maintained after passage through a freeze/thaw cycle (Fig.S1). Furthermore, RNAseq analysis revealed that exAM showed high general expression levels of AM specific core macrophage genes 3 compared to other tissue resident macrophage populations such as peritoneal macrophages (Fig.2G). This was reflected in high expression levels of AM specific macrophage transcription factors genes ${ }^{3}$, including Pparg, Car4, Clebpb and Bhlhe41 (Fig. $2 \mathrm{H}$ ), which have been shown to be important regulators of AM identity and self-renewal $\underline{\underline{2}} \underline{20} \underline{21} \underline{22} \underline{23}$. Together these results showed that exAM not only maintained macrophage functional characteristics but also AM specific gene expression through long term culture.

\section{The exAM transcriptome shows adaptations to the culture environment}

The microenvironment has emerged as a potent determinant of macrophage gene

176 expression $\underline{2}$. For example, the transfer of mouse $\underline{\underline{9}}$ or human $\underline{10}$ microglia from the brain environment to ex vivo culture results in major changes in gene expression and AM in culture show significant changes in cytokine responsiveness and glucose metabolism compared to in vivo conditions $\underline{\underline{11}}$. We therefore wondered whether the culture environment also imposed changes in gene expression on exAM, despite the observed overall conservation of macrophage function and phenotype (Fig.2). number of significant transcriptional changes (FDR 0.05) with 1370 genes up-regulated more 
than 2-fold and 376 genes upregulated more than 10-fold, whereas 2317 genes were downregulated more than 2 -fold and 857 genes were down-regulated more than 10 -fold (Fig.3A).

We hypothesized that some of these changes in gene expression might be related to the expansion capacity in culture. Indeed, consistent with the observations in Fig.1B we found enrichment of GO term cell cycle gene sets and increased expression of cell cycle regulators (Fig.3B). We also found enrichment and overexpression of TGFbeta signaling related genes, consistent with reports that AM self-renewal depends on an autocrine TGFbeta signaling loop $\underline{24}$ (Fig.3C).

Although exAM maintained overall similar high expression levels of core AM specific genes $\underline{3}$ as $A M$ in vivo, compared to other resident tissue macrophage populations (Fig.2G), there appeared to be reductions in some genes. To investigate this in more detail we analyzed the individual expression levels of AM core genes and observed that whereas the majority showed no or low changes in gene expression, several genes were substantially downregulated. These genes included surfactant proteins, lipid metabolism genes and Epcam, a homotypic adhesion molecules of the CAM family (Fig.3D ,Fig.S2). This is consistent with the loss of the natural alveolar niche environment in culture, where these genes might be obsolete or lack appropriate induction cues.

To analyze the nature of these changes in more detail we performed k-means clustering. Whereas the majority of expressed genes showed no significance difference between culture and in vivo conditions, we identified two smaller clusters of up- and down-regulated genes, respectively (Fig.3E).

Pathway analysis of the up-regulated cluster revealed cytoskeletal, adhesion and migration pathways, consistent with adaptation to the different surface properties of the culture environment (Fig.3F,G). Furthermore, oxygen response, amino acid and glucose metabolism pathways (Fig.3F,G and Fig.S2) were upregulated, indicative of the altered oxygen pressure, the availability of nutrients in culture and the upregulation of glycolytic pathways in culture that are restricted in vivo $\underline{\underline{11}}$.

Pathway analysis of the down-regulated cluster revealed predominantly immune system pathways, including bacterial and viral response, inflammatory and antigen presentation terms. This is consistent with the fact that the alveolar macrophages were taken from a barrier tissue constantly exposed to environmental microbes to a sterile tissue culture environment (Fig.3F,G). long term culture, a significant number of genes are sensitive to lost or gained cues in culture that regulate the interaction of AM with their microenvironment. 
Based on the observed transcriptional adaptations to the culture environment we wanted to determine whether these changes were stable or reversible. We therefore employed an intratracheal transplantation protocol to establish whether full transcriptional identity of AM could be restored, once the microenvironmental cues of the natural niche in vivo were provided again. Several protocols have been previously used to transfer different macrophage populations into the alveolar space of the lung. These protocols typically involve transplantation into an empty niche, from which the resident AM have been depleted genetically, chemically or by irradiation $\underline{7} \underline{21} \underline{25} \underline{26}$. Since these procedures all involve strong disturbance of tissue environment and homeostasis, we considered them not ideal to investigate the effects of the natural niche environment on exAM identity in vivo. Here we therefore established a transplantation protocol into an unmodified niche of wt mice. To control for the effects of transplantation itself we compared transplanted fresh AM from BAL (tAM) and transplanted ex vivo amplified exAM (texAM) to the resident host AM populations (Fig.4A). Using this protocol, we could demonstrate stable long-term contribution of transplanted cells to the AM pool for at least 4 months with highly similar contribution rates of $5-15 \%$ for both tAM and texAM (Fig.4B). Furthermore, FACS analysis demonstrated undistinguishable expression levels between all three populations for the general myeloid and macrophage markers CD11b and CD64 as well as for the AM specific markes CD11c and SiglecF (Fig.4C). Together this showed that despite extended passage time in culture, exAM maintained the capacity of long- term contribution to the AM pool with normal stable phenotype and self-renewal in vivo.

In order to further determine whether the global transcriptome could be restored to the in vivo state upon transplantation of exAM from the culture environment to the natural niche in vivo, we investigated the similarity of the different AM populations by RNAseq. Spearman's correlation analysis revealed that the differences of cultured ex AM to in vivo samples disappeared upon transplantation. TexAM showed a high degree of correlation to the other two in vivo samples, both tAM and host AM populations (Fig.4D). This was further confirmed by comparison to published data sets from other tissue resident macrophage populations using PCA analysis. As shown in Fig.4E, texAM were indistinguishable from tAM or resident host AM but different to all other resident macrophage populations. As an indication of the high degree of similarity, our samples were closer to each other than to published AM data sets from another lab generated with a different protocol (Fig.4E).

In a more precise analysis of changes in gene expression between tAM and texAM, we only detected 56 genes that were significantly expressed with more than twofold change (FDR: 0.05). This contrasted with 3547 differentially expressed genes between exAM in culture and 266 after transplantation (texAM) (Fig.4F,G). A similarly high number of 3687 differentially 
expressed genes were detected when comparing exAM to host AM, which were reduced to only 217 differentially expressed genes after transplantation. When analyzing whether any of the genes that differed between texAM and tAM or host AM, only very few genes overlapped (Fig.2H), indicating that likely none of the other differences were due to the retention of culture specific genes expression in vivo, but to experimental noise or transplantation specific effects, as similar differences as for texAM versus host AM were also detected between tAM and host AM (Fig.S3)

Finally, the analysis of the core AM signature genes showed that the few genes related to lipid metabolism, surfactant and adhesion molecules that were lost in the culture environment (Fig.3D) were fully restored after transplantation of exAM into their natural niche (texAM) with nearly no detectable differences to the other in vivo AM samples (Fig.4I). Similar observations were made for the few AM specific genes that had been upregulated in culture (Fig.4l).

Together this showed that the transcriptomic adaptations of long-term cultured exAM to the culture environment were fully reversible and could be fully restored to the characteristic signature of resident AM upon exposure to the cues of the natural niche environment in vivo.

\section{Epigenetic changes of exAM in culture are fully restored in vivo}

The full restoration of transcriptomic identity upon transplantation of exAM into the natural alveolar space and the complete loss of the transcriptional adaptions to the culture environment opens the question as to the underlying epigenetic regulatory mechanisms. We therefore performed ATACseq analysis to determine the status of chromatin accessibility during these transcriptional changes. As one potential explanation of the complete reversibility of transcriptional changes we considered the possibility of unchanged chromatin accessibility between in vivo and culture conditions. The comparison of open chromatin regions (OCR) by ATACseq in cultured exAM and BAL AM in vivo, however, revealed a substantial number of differentially closed (723) or accessible (1504) OCR between the two conditions (Fig.5A), and thus excluded this explanation.

GO term analysis of the genes associated with these OCR alterations yielded similar terms of adaptation to the cell culture environment as the transcriptomic analysis. Whereas terms for metabolism, cellular proliferation, cell adhesion and migration were enriched, immune response terms were depleted in culture (Fig.5B). As individual examples of this, peaks not present in fresh BAL AM were induced in culture in the regulatory regions of cyclin D1 and $\mathrm{mdm} 2$, two cell cycle genes, of Sphk1 and Pkp2, two genes important for cell adhesion, and of HK1 and HK2, critical regulators of glycolysis. Similarly, OCR of several genes important in lipid metabolism (Acaa1b and Cidec) and in the immune response, such as several MHCll genes (H2-Aa, H2-Ab1, H2-Eb1) disappeared in exAM culture (Fig.5C). 
309 Environmental cues can induce long- lasting epigenetic changes despite full reversibility of the 310 transcriptional alterations induced by such signals. For example, we have shown previously that LPS stimulated hematopoietic stem cells (HSC) show transcriptional changes that quickly come back to normal but conserve a long-term epigenetic memory of the stimulus 27 . We therefore wondered whether culture-induced epigenetic changes in exAM were conserved upon re-transfer into the natural environment in vivo. In order to address this question, we used the same transplantation protocol as before (Fig.4A). Strikingly, culture specific alterations in chromatin accessibility were fully restored upon transplantation of exAM into the natural alveolar niche. This was indicated by nearly identical OCR in transplanted cells (texAM) compared to resident AM of the recipient (host AM), whereas the comparison of cells in culture (exAM) showed the same difference to host AM as to freshly isolated BAL (Fig.5A). From the over 2500 differential OCR detected between AM in culture and in vivo (exAM versus host $A M)$, only 15 were conserved after re-transplantation of exAM into the natural niche (texAM versus host AM, Fig.5D). Consistent with this, the comparison of exAM to texAM showed similar differences, whereas BAL and host AM showed a nearly identical OCR profile (Fig.S4). The similarity of all in vivo samples among each other, including transplanted expanded AM (texAM) and the similar strong differences of all in vivo samples to exAM in culture was also indicated by a heat map of unsupervised cluster analysis (Fig.S5). Importantly, analysis of individual OCR showed that the culture-induced peaks in regulatory regions of cell cycle, adhesion or glycolysis genes were lost again, whereas peaks in immune response genes that were lost in culture were re-established, when cultured cells (exAM) were transplanted into the natural in vivo environment (texAM) (Fig.5C).

Analysis of transcription factor binding motifs associated with differential OCR between culture and in vivo samples revealed that besides ATF3 and KLF motifs, binding sites for the core myeloid PU.1, C/EBP and Runx transcription factors were associated with both the differential open and closed OCR (Fig.S6), suggesting that the changes in chromatin accessibility might occur on pre-existing myeloid enhancer platforms.

Finally, analysis of regulatory regions for core AM specific transcription factors and surface markers identified AM specific OCR that were not detected in other myeloid cells or other cell types of the immune system, identifying them as markers of epigenetic AM identity (Fig.5E).

341 For example, AM specific peaks of the transcription factor PPARg $\underline{18}$, a key regulator of AM identity, are not detected in other macrophage populations or immune cells, whereas the surface marker CD11c (Itgax) that is expressed in AM and other cell types shows AM specific and general OCRs. Importantly, these key regulatory regions of AM identity did not change across samples and were equally present in $A M$ in vivo, after expansion in culture (exAM) and upon re-transplantation in vivo (texAM), indicating that epigenetic AM identity was not lost in culture. 
Together the analysis of epigenetic chromatin accessibility indicates that AM identity was conserved through culture expansion and that adaptations to the culture environment were transient, reversible and fully restored to the in vivo status upon transplantation into the alveolar niche.

\section{Expanded alveolar macrophages show full long-term reconstitution of alveolar macrophage niche in vivo}

Given the full restoration of transcriptional and epigenetic identity of exAM in vivo, we wondered whether they were also functionally capable of reconstituting an empty alveolar macrophage niche. Since transplantation into the full niche of wt mice only allows a small contribution of donor cells (Fig.4A,B), we took advantage of GM-CSFRbeta KO (csf2 $\mathrm{rb}^{-/}$) mice, which are deficient for alveolar macrophages and can be transplanted with different sources of monocytes or macrophages $\underline{7} \underline{8} \underline{25} \underline{28} \underline{29}$. We therefore transplanted CD45.1 exAM long term

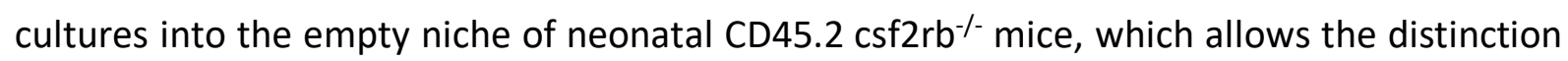
of donor and recipient cells. Interestingly, 8 months after transplantation we detected nearly $100 \%$ of CD45.1 donor cells with a SiglecF+/CD11c+ AM phenotype in transplanted csf2 $\mathrm{rb}^{-/}$ mice, the same proportion as in CD45.1 WT mice of the same age, whereas untransplanted csf2 $\mathrm{rb}^{-1-}$ mice showed no CD45.1 cells or SiglecF+/CD11c+ AM (Fig.6B,E,F). Transplanted and WT control cells showed the same morphology by brightfield microscopy and staining with Kwik-diff dye of cytospin samples (Fig.6C). We also obtained the same absolute number of cells from the BAL fluid of transplanted csf2 $\mathrm{rb}^{-/}$mice as from CD45.1 WT control mice (Fig.6G). This demonstrated that long term cultured exAM were capable of full long term reconstitution of an empty AM niche in vivo. A similar full reconstitution was also already seen 3 months after transplantation (Fig.6E-H, Fig.S7), demonstrating a similar potency of seeding the niche in vivo as fetal monocytes, the most prominent source of populating the lung in normal development $\underline{\underline{7}} \underline{\underline{30}}$.

Due to the complete absence of AM, $\mathrm{csf} 2 \mathrm{rb}^{-/-}$mice show a pulmonary alveolar proteinosis (PAP) pathology that is characterized by accumulation of mucus and debris in the alveolar lung fluid. Microscopic inspection (Fig.6C) or FACS analysis (Fig.6D,H) demonstrated a strong reduction of debris in the transplanted lungs (Fig.6D,H) that in some cases showed complete clearance of debris similar to WT control mice (Fig.6 C,D,H). Thus, in culture expanded exAM maintained the key homeostatic function of AM to clear alveolar mucus.

Together these results showed the capacity of expanded AM in culture (exAM) to functionally repopulate an empty alveolar niche in vivo and to self-maintain homeostatic numbers long term. 
Here we have shown that alveolar macrophages can be maintained through long term culture and can be expanded massively without losing tissue resident macrophage identity. Although significant epigenetic and transcriptional culture adaptations occur, they are fully reversible upon transplantation into the natural alveolar niche in vivo (Fig.7). This is conceptually important as it indicates a robust cell endogenous epigenetic setup that is stable through many cell divisions and provides the flexibility to adapt to different environmental cues without losing identity. In practical terms, expanded alveolar macrophages provide a nearly unlimited source of genetically unmodified and untransformed normal macrophages and a new cell culture system that provides the convenience of a cell line but the ability to quantitatively repopulate the natural niche in vivo with full restoration of epigenetic and transcriptomic identity. The potential to shuttle cells between ex vivo culture and natural niche in vivo provides a unique experimental system for screening and in vivo validation approaches or combined large-scale biochemical and genetic investigation in an untransformed cellular system with direct in vivo relevance.

Cell culture has had enormous impact on the progress of biological research ranging from cancer to stem cell research $\underline{31}$. In immunology it has boosted vaccine development, the generation of monoclonal antibodies and cellular therapy and has been essential for many fundamental discoveries. Despite this general usefulness it has been put into question how close cells in culture are to their in vivo equivalents due to the loss of essential in vivo cues and new culture stimuli not encountered in vivo. For example, mouse and human microglia cells in culture undergo major changes in gene expression and loose in vivo functionality $\underline{9} \underline{10}$ 12. Similarly, AM show significant changes in cytokine responsiveness and metabolism when put in culture and it has been suggested that cultured macrophages do not reflect macrophage identity in vivo $\underline{11}$. Indeed, here we also observed significant changes in gene expression related to the adaptation to the culture environment, including for genes involved in cellular adhesion/migration, proliferation and metabolism. This was in particular the case for genes related to glycolysis, consistent with the high availability of glucose in the culture medium compared to the low levels in the alveolar niche and the shift to glycolytic energy metabolism of $A M$ in culture $\stackrel{11}{ }$. We also observed changes in adhesion molecules and cytoskeletal genes, which is consistent with the different surfaces and general physical cues that AM encounter in the culture dish compared to the natural alveolar niche. Furthermore, immune response related gene expression was lost in culture, which might be explained by the loss of the stimulation from the microbiome of the lung mucosal surface in the sterile culture environment. Importantly, however, both positive and negative changes in gene expression were reversible and fully restored upon transplantation into the natural alveolar niche in vivo, confirming a potent role of the microenvironment as determinant of macrophage gene expression $\stackrel{2}{ }$. 
The interesting question emerging from these observations is how AM conserve the ability to respond to these different environmental cues. We observed that the changes in gene expression were also accompanied by changes in chromatin accessibility. Differentially open and closed OCR in culture were similarly associated with adhesion, proliferation, metabolism and immune response terms, respectively (Figs. 5,7$)$. Interestingly, both differentially opened and closed OCR were enriched for binding sites of PU.1, C/EBP and Runx (Fig.S6), transcription factors that determine macrophage identity $\underline{32}$. It has been suggested that these factors establish a core macrophage enhancer platform on which additional signal induced transcription factors converge ${ }^{33} 34$. Consistent with this, culture induced OCR were also enriched for ATF3 and KLF transcription factor binding sites, which are typical signal induced factors. Together this suggests that a stable enhancer architecture maintains cellular identity through culture, on which accessory transcription factors can activate environment specific gene expression. Consistent with this, the expression of core macrophage and alveolar macrophage specific transcription factors like Runx2, Car4, Pparg, C/ebpb and Bhlhe41 (Fig.2H) and downstream AM specific genes (Fig.2G,4I) as well as AM specific surface markers (Fig.2E) were maintained through culture. Furthermore, core AM specific transcription factors and surface markers showed maintained OCR in their regulatory regions (Fig.5E). This could explain how highly stable AM specific identity is maintained through culture, while allowing a spectrum of responsiveness to environmental cues in vivo and in culture with the corresponding repertoire of adapted gene expression. In more general terms this might also explain the high degree of plasticity of macrophages in responding to a diverse set of stimuli 35 .

The observed full restoration of normal chromatin accessibility in vivo, despite the significant epigenetic changes acquired in long term culture, is distinctly different from scenarios of trained immunity. Although in principle both resident $\frac{36}{6}$ and monocyte-derived $\underline{21} \underline{26}$ AM also appear to be capable of trained immunity, the underlying mechanism and inducing signals might be different. For example, we have shown previously that LPS stimulation of HSC induced only short-term transcriptional changes but long-term epigenetic memory through a TRIF and C/EBPb signaling axis프. Perhaps, in this case, C/EBPb acts as a pioneering factor establishing new enhancer landing sites for other transcription factors, whereas in the case of AM these enhancer assemblies are already present in vivo and maintained through culture. Beta-Glucan stimulation can also induce trained immunity through epigenetic changes in monocytes in an Akt, mTOR and glycolysis dependent manner 37 38 39 . Interestingly, PI3K/Akt and mTOR pathways as well as glycolysis were also induced in exAM (Fig.3F) but changes in chromatin accessibility were completely restored after re-transplantation in vivo. This indicates that these pathways are necessary but not sufficient for the induction of epigenetic memory in myelo-monocytic cells.

Our results are important on three fronts: conceptually, for their therapeutic potential and as experimental model. 
472 Our observations extend our previous findings that demonstrated the compatibility of self473 renewal with the differentiated state of mature macrophages $\underline{17} \underline{18} \underline{40}$. Here we show that AM 474 can be expanded extensively in culture for almost a year or more, but still assume full 475 epigenetic identity and functionality upon re-integration in their natural environment in vivo. 476 This is conceptually exciting, because it demonstrates that neither culture adaptations nor 477 long term proliferation erase the epigenetic and transcriptional identity of AM. Such behavior 478 is normally only known for stem cells that can be expanded extensively or indefinitely in 479 culture and still functionally integrate into a tissue environment, for example pluripotent stem 480 cells that can integrate into an embryo and give rise to a whole new organism or epithelial stem cells that can provide functional skin tissue for transplantation $\underline{31} \underline{41}$.

Our results are therefore also highly promising for all potential future applications of macrophage cellular therapy. T-cells have been highly successful in cancer immune-therapy $\underline{15}$. Despite their potential for cancer therapy, the use of macrophages for such applications, has so far been limited by the difficulty to grow large numbers of macrophages in culture $\underline{16}$, as has been possible for T-cells $\underline{15}$. Our results now demonstrate that in principle large scale and longterm expansion of macrophages ex vivo is possible without compromising the cells' identity in vivo. The robustness of macrophage identity through culture could also be important for macrophage based cellular therapies in respiratory disease such as COVID19, where macrophages and the relative contributions of monocyte-derived and resident alveolar macrophages play important roles in disease development $\underline{42}$.

Finally, we show that expanded AM are a long-term reliable culture model for resident macrophages that provides similar advantages as a cell line, in terms of providing large quantities of cells and the possibility of starting and stopping cultures by freeze/thawing without being dependent on complex differentiation protocols. In addition, the possibility to shuttle between in vivo and culture environment provides a unique experimental system for genetic and biochemical manipulation that cannot be provided by other macrophage culture

\section{0 systems.}

501

\section{Acknowledgements}

503

This study was supported by institutional grants from TU Dresden and the Institut National de la Santé et de la Recherche Médicale, Centre National de la Recherche Scientifique, and AixMarseille University and grants to M.H. Sieweke from the Agence Nationale pour la Recherche 507 (ANR-17-CE15-0007-01 and ANR-18-CE12-0019-03), Fondation ARC pour la Recherche sur le 508 Cancer (PGA1 RF20170205515), and the European Research Council (ERC) under the European 509 Union's Horizon 2020 research and innovation program (grant agreement number 695093 510 MacAge). SS received a doctoral fellowship from the MDC. M.H. Sieweke is an Alexander von 511 Humboldt Professor at TU Dresden. The authors declare no competing financial interests. 


\section{Animals}

516 C57BL/6 CD45.1+ and CD45.2+ mice were obtained from Charles River and Janvier, 517 respectively. The csf2 $\mathrm{rb}^{-/}$mice were kindly provided by Dr. Melanie Greter (University of 518 Zurich) and described previously $\underline{43}$. Age of donor and recipient mice were between 6 and 8 weeks old. Mice were housed under specific pathogen-free conditions in individually ventilated cages in a controlled 12-hour reverse light/dark cycle and were provided with food and water ad libitum. In vivo procedures were performed following the protocols approved by the ethics committee of $n^{\circ} 014$, Marseille, France (APAFIS\#3292-2015122109359224) and the TU Dresden and the Landesdirektion Sachsen in accordance with institutional, national and European animal welfare legislation (TVV28/2018 AKZ: IC 114-G0160/16 and DD24.1$5131 / 449 / 30)$.

526

\section{isolation and ex vivo expansion of alveolar macrophages (AM)}

Isolation of AM from BAL was done as previously described $\underline{19}$. The expansion and maintenance of exAMs ex vivo was done as described previously $\underline{\underline{19}}$. Briefly, cells were plated at a density of $1.1-1.5 \times 10^{6}$ per $10 \mathrm{~cm}$ petri dish in $10 \mathrm{ml}$ complete medium. The medium was supplemented with murine $2-5 \%$ GM-CSF supernatant from J558L cells transfected with murine GM-CSF.

\section{Preparation of lung homogenates for flow cytometry analysis}

Mice were euthanized by cervical dislocation, lungs were collected, cut in small pieces and incubated with $1 \mathrm{mg} / \mathrm{ml}$ collagenase-2 (Worthington) and $0.15 \mathrm{mg} / \mathrm{ml}$ DNasel (Sigma) at $37^{\circ} \mathrm{C}$ for $30 \mathrm{~min}$ with constant agitation. Cell suspension was filtered through a $70 \mu \mathrm{m}$ mesh and erythrocytes were removed by RBC lysis (RBC Lysis buffer, Invitrogen).

\section{Flow cytometry}

Lung cell suspensions was pre-incubated with Fc receptor blocking antibody (clone 2.4 G2, BD Pharmingen or TruStain FcXTM, Biolegend) and Zombie fixable cell viability dye (NIR, Aqua or UV, Biolegend) or DAPI (Sigma) for $15-20 \mathrm{~min}$ at $4^{\circ} \mathrm{C}$. For antibody staining, cells were incubated with antibody cocktail for $20 \mathrm{~min}$ at $4^{\circ} \mathrm{C}$. FACS sorting and analysis was done with FACSCanto, LSRII and LSRFortessa systems (BD). The following antibodies were used for staining cells: anti-CD11b (clone M1/70, eBioscience or Biolegend), anti-CD11c (clone N418, eBioscience or B-Ly6, BD), anti-F4/80 (clone BM8, eBioscience), anti-MHCII (clone M5/114.15.2, eBioscience), anti-CD45.1 (clone A20, eBioscience or Biolegend), anti-CD45.2 (clone 104, eBioscience or Biolegend), anti-Ly6C (Clone AL-21, BD), anti-CD64 (clone X545/7.1, Biolegend or BD), anti-SiglecF (clone E50-2440, BD). Diva software was used for acquisition of data and Flowjo software V10 for data analysis (TreeStar). 
Mice were anesthetized by isoflurane inhalation, placed on their back, the tongue was gently pulled out and $0.8-1 \times 10^{6}$ freshAM or exAMs in $80-100 \mu$ I PBS were instilled intra-tracheally using $1 \mathrm{ml}$ syringe with a blunt 22G gavage needle. Mice were observed while recovering from anesthesia and then returned to their cages for routine care and handling. Neonatal Csf2rb $\mathrm{r}^{-1}$ mice (postnatal day 3 ) were anaesthetized with isoflurane and transplanted with $0.4 \times 10^{5} \mathrm{AM}$ resuspended in PBS and in a total volume of $7 \mu$.

\section{Ex vivo alveolar macrophage assays}

\section{Cytospins and in-well photography}

AM were plated at a density of approx. $0.4 \times 10^{5}$ cells $/ \mathrm{cm}^{2}$ for 4 hours for attachment at either 6-well or $100 \mathrm{~mm}$ non-treated cell culture dishes. Bright-field images acquired from well center using Zeiss Axio Vert A1 microscope at 10x magnification. Fresh BAL AMs or exAM (2 months culture, detached and counted) were subjected to cytocentrifugation $300-450 \mathrm{rpm}$ for $4 \mathrm{~min}$ using a Cytospin 4 (Thermo Fisher) and performed Diff-Quik staining following manufacturer's protocols (9990700, Thermo Scientific ${ }^{\mathrm{TM}}$ Rapid-chrome $^{\mathrm{TM}}$ Kwik-diff $^{\mathrm{TM}}$ kit). Images were acquired using the 3DHistech slide scanner and images were processed using Qupath v.0.2.0 with built-in Image J image analysis software. For Csf2rb-/- transplants, brightfield images were taken with an inverted microscope (Dmi1, Leica) after transfer of BAL cells to a Neubauer counting chamber. Cell suspensions were cytospin at $800 \mathrm{rpm}$ for $3 \mathrm{~min}$ using a Cytospin 4 (Thermo Fisher), followed by Kwik-diff staining according to the manufacturer's instructions (9990700, Thermo Scientific ${ }^{\mathrm{TM}}$ Rapid-chrome $^{\mathrm{TM}}$ Kwik-diff $^{\mathrm{TM}}$ kit). Images were taken with a Zeiss Axiolab A1 microscope.

\section{Cell cycle analysis}

AM were plated at density of $0.2 \times 10^{6}$ cells/well of non-treated 12 -well dishes (Nunc). The cells were directly pulsed with $10 \mu \mathrm{M}$ 5-ethynyl-2'-deoxyuridine, EdU (C10634, Thermofischer) for 4 hours at $37^{\circ} \mathrm{C}$ to label proliferating cells. Cells were then detached, washed and preincubated with Fc receptor blocking antibody and cell viability dye followed by antibody staining as described under flow cytometry section. Cells were fixed with 4\% PFA for 20 min at $4^{\circ} \mathrm{C}$ and permeabilized with Click-iT saponin permeabilization and wash buffer for 15 minutes at $4^{\circ} \mathrm{C}$. Click-IT reaction was performed using the Click-iT Plus EdU Alexa Fluor 647 Flow Cytometry Kit (C10634, Thermofischer) as per manufacturer's instructions. For DNA staining, cells were resuspended in permeabilization buffer containing $1 \mu \mathrm{g} / \mathrm{ml}$ DAPI for 30 minutes at RT. FACS data acquisition was done in the BD LSR Fortessa and analysis using FlowJo V10.

\section{Phagocytosis assay using fluorescent latex beads}

592 AM were incubated in growth media containing Fluorescent yellow-green, carboxylate593 modified polystyrene Latex beads (L4655, Sigma-Aldrich) at 1:400 dilution for 1 hour at 370 C. 594 Supernatant medium containing beads were discarded post-centrifugation, washed three 
times (5 min each) in PBS. After a brief cytocentrifugation, the cells were fixed using 4\% PFA for $10 \mathrm{~min}$ and permeabilized with $0.1 \%$ Triton-X in PBS for 15 min at RT. Cells were incubated in blocking buffer ( $5 \%$ donkey serum+1\%BSA in PBS) for $15 \mathrm{~min}$ at RT followed by Actin red staining ( $2 \mathrm{drops} / \mathrm{ml}$ in PBS). All the steps were carried out with adequate washing steps (3 times, 5 min each using PBS). The slides were air-dried and mounted with ProLong ${ }^{\mathrm{TM}}$ Gold Antifade mountant (P36931, Thermo Fischer).

601

602

\section{Phagocytosis assay by flow cytometry}

603

AMs were plated for 4 hours and replaced with fresh medium containing $2 \mu \mathrm{g} / \mathrm{ml} \mathrm{pHRhodo} \mathrm{TM}^{\mathrm{TM}}$ Red Zymosan Bioparticles ${ }^{\mathrm{TM}}$ Conjugate (P35364, Thermo Fischer) and incubated at $37^{\circ} \mathrm{C}$ and for controls at $4^{\circ} \mathrm{C}$ on ice for 2 hours. Subsequently, AMs were detached and processed for flow cytometric analysis.

607

\section{Detection of lysosome and lysosomal active cathepsin B}

AMs were incubated with Acridine orange (1:1000 of stock solution in PBS, 6130 - Bio-Rad) for $20 \mathrm{~min}$ at $37^{\circ} \mathrm{C}$ or with Magic Red solution ( $1 \mathrm{x}$ final concentration, ICT937 - Bio-Rad) for 50min at $37^{\circ} \mathrm{C}$. Nuclei stained using Hoechst 33342 solution (1:200, 62249 - Thermo Fischer) for $10 \mathrm{~min}$ at $37^{\circ} \mathrm{C}$. After staining, AMs were mounted on slides and immediately imaged using Zeiss inverse ApoTome microscope and analyzed using FIJI image analysis software.

614

\section{Detection of LPS induced ROS generation}

AMs were stimulated with $2 \mathrm{mg} / \mathrm{ml}$ E.coli O55:B5 LPS (L4005, Sigma) overnight. The next day, AMs were detached and incubated with $100 \mu \mathrm{M}$ CM-H2 DCFDA (1:100, C6827 - Thermo Fischer) for $20 \mathrm{~min}$ at $37^{\circ} \mathrm{C}$. The nuclei were stained using Hoechst 33342 solution (1:200, 62249 - Thermo Fischer) for $10 \mathrm{~min}$ at $37^{\circ} \mathrm{C}$. After staining, the AMs were mounted on slides and imaged immediately using Zeiss inverse ApoTome microscope and analyzed using FIJI image analysis software.

622

\section{RNA sequencing.}

624 For expanded AMs (exAM), total RNA was extracted from 3 months culture of exAMs. In vivo AM subsets, were sorted (Live, Singlets, SiglecF+CD11c+, CD45.2+ or CD45.1+) from total lung homogenates directly into RNA lysis buffer (RLT buffer, Qiagen) for RNA extraction using BD FACSArialll machine. Each sample was generated from a pool of 3 mice in duplicates. Amplified cDNA was prepared from 2 ng of total RNA using the Ovation RNA-seq system V2 (NuGEN Technologies, Inc.) following manufacturer's instructions. Briefly, first-strand cDNA was prepared using a combination of random and poly-T DNA/RNA chimeric SPIA (single primer isothermal amplification) primers and reverse transcriptase. Priming sites were used to synthesize second strand cDNA using a DNA polymerase, was purified using Agencourt

633 RNAClean XP beads (Beckman Coulter Inc.). and subjected to SPIA. Amplified cDNA was 634 purified using AMPure XP beads (Beckman Coulter Inc.) and $500 \mathrm{ng}$ was fragmented by sonication using a Covaris E210 instrument. Library preparation was performed using Ovation 
636 Ultralow Library System kit (NuGEN Technologies, Inc.) according to manufacturer's

637 instructions. Briefly, $100 \mathrm{ng}$ of amplified cDNA was blunted, phosphorylated and ligated to 638 indexed adapter dimers. The libraries were then enriched by PCR amplification and surplus

639 PCR primers were removed by purification using AMPure XP beads. DNA libraries were 640 checked for quality using 2100 Bioanalyzer (Agilent) and quantified using Kapa Sybr Fast Light 641 Cycler 480 qPCR Kit (Kapa Biosystems) following manufacturer's instructions.

\section{ATAC sequencing.}

644 BAL AM pooled from 3 mice were expanded ex vivo for 2 months and i.t transplanted into 645 lungs of 2-3 WT CD45.1 mice. The lungs were collected at 4 months post transplantation and 646 sorted for CD45.2+ exAM or CD45.1+ host AM using BD FACSArialll machine. $10 \times 10^{3}$ cells 647 from 3 mice each of BAL AM, exAM, texAM or Host AM were pooled and used for ATAC-seq. 648 Fast ATAC-seq protocol was used as described ${ }^{44}$. Cells were lysed with $1 \%$ of digitonin in TD 649 buffer and subjected at the same time to Tn5 transposase (Nextera). The reactions were 650 incubated $30 \mathrm{~min}$ at $37^{\circ} \mathrm{C}$. DNA were purified using Cleanup Minelute kit (28204, Qiagen) 651 according to the manufacturer protocol. Library were prepared using Nextera DNA Library 652 Preparation Kit (FC-121-1030, Illumina) with a 4 cycles PCR according to the manufacturer 653 protocol. DNA was quantified with a quantitative-PCR using Nextera primer and amplified with 654 a second PCR of 12 to 18 cycles. Libraries were cleanup with a ratio of 0,7 of AMPure XP beads 655 (Beckman coulter) and quantified with a Qubit fluorometer (Life Technologies). Library quality 656 was assessed by using 2100 Bioanalyzer (Agilent technologies). Libraries were sequenced with 65775 paired-end using NextSeq 500 instrument (Illumina) for an average of $1 \times 10^{7}$ reads per 658 sample (to verify).

659

\section{Bioinformatic analysis.}

662

The following analyses were done with $\mathrm{R}$ (v3.3). Samples were aligned to mouse genome version mm10 using Bowtie aligner. $A$ table of raw counts was generated using htseq-count and $\mathrm{R}$. All samples were normalized to $1 \mathrm{M}$ reads using the cpm function from the edge $\mathrm{R}$ package. In order to check reliability of samples replicates, we conducted PCA analysis on normalized expression data on all expressed genes (excluding lowly expressed genes) and correlation tests on normalized expression data. For Identification of differentially expressed genes, a filtering step was conducted depending on the comparison of interest. Genes having more than $4 \mathrm{cpm}$ in at least one condition in a pairwise comparison were kept for the remaining analysis. For detection of differentially expressed genes, raw data were treated using DESeq package. GO analyses were done on differentially expressed genes after k-means clustering between exAM vs Host with help of ChIPseeker package. $\mathrm{R}$ software was used to generate heatmaps.

674

675

676 
678 Data were pooled from 3 independent experiments. Fastq files quality control were done by 679 fastqc tool. Adapter trimming was done using Cutadapt. Bowtie2 was used to align and index 680 sequences on the mm10 mouse genome and thus generated the first series of bam files. Then, 681 Peaks detection was made on these bam files by Genrich. Then, from the first series of bam 682 files, a second series was generated as following: after using Bowtie2, mitochondrial DNA was 683 removed with Samtools. Picard was used to remove PCR duplicates. Finally, Samtools allowed 684 to remove non-unique alignments. Bigwig files were generated from bedgraph files thanks to 685 bdg2bw script (https://gist.github.com/taoliu/2469050). These bedgraph files were created 686 from the second series of bam files. All peaks detected by Genrich from exAM, texAM, Host, 687 BAL samples were pooled and merged together with bedtools ( $v$ 2.26.0). Then a table of 688 counts was generated by bedtools from these peaks. Signal was computed from the second 689 series of bam files. Then peaks with more than 10 counts in at least one condition were kept 690 for the following analysis. Opened and closed peaks were detected with the DESeq package. 691 Peaks with adjusted $p$-values less than 0.05 were declared as differentially open between two 692 conditions. Comparisons were texAM vs host AM, exAM vs host AM, exAM vs texAM. Then $693 \log 2$ (count+1) were represented in scatterplots comparing condition two by two. Peaks with $694 \log 2$ FC computed by DESeq2 more than 1.5 (resp. less than 1.5) were highlighted in red (resp. 695 in blue).

696 For Motif enrichment analysis, 1492 peaks were selected as gained in exAM (as compared to 697 host) and 937 were selected as lost. Then motif analysis was performed on these two groups 698 of peaks using HOMER (http://homer.salk.edu/homer). HOMER, inter alia, is a discovery motif 699 analyzer and screens for enrichment of known motifs. HOMER perl script 700 findMotifsGenome.pl was used with the mm10 mouse genome as a background (random 701 genomic sequences samples according to GC content of input sequences). Venn diagrams 702 were plotted using VennDiagram package. GO analysis and Genes association with peaks were 703 done with GREAT tool (v4.0.4) using default options to assign regions and using minimum 704 region-based fold enrichment of 1.2 and term annotation counts (5-1000) were set as global 705 controls for GO term analysis. For peak visualization reference ATAC-seq sample bigwig files 706 were downloaded from the Immgen consortium ${ }^{45}$ (GSE100738). peak visualization on our 707 samples independently or in parallel with the Immgen references using integrative genomics 708 viewer (IGV, version 2.10.2) and were auto scaled by group. 


\section{Figure1. Alveolar macrophages show a massive expansion potential in long term culture}

a) Growth curve of AM in liquid culture represented as cumulative population doublings over time. Linear regression of exponential population growth (Orange line) with $95 \%$ confidence intervals (Black lines). Data shown are from biological quadruplicates and represented as Mean \pm SD. Inset: Schematic of alveolar macrophages (AM) isolation by broncho-alveolar lavage (BAL) and ex vivo expansion culture (exAM).

b) Cell cycle analysis on freshly isolated AM or exAM (3.5 months), (Live, singlets, SiglecF+, CD11c+ cells) using EdU $(10 \mu \mathrm{M}, 4$ hours) for proliferating cells and DAPI for DNA-content as evaluated by flow cytometry. FACS plots are representative of biological quadruplicates. Data is represented as Mean \pm SD.

Figure2. Expanded alveolar macrophages maintain characteristic phenotype and function in long term culture

(a-c) Representative images of plated BAL AM or exAM (2 months) ex vivo.

a) Brightfield-images (10x magnification) and Kwik-diff histological staining on cytospins. Scale bar represents $20 \mu \mathrm{m}$ and $10 \mu \mathrm{m}$ for magnified cell examples. Data shown are representative of two independent experiments.

b) Representative fluorescence staining for the lysosomal marker acridine orange (AO) and lysosomal cathepsin B activity using magic red (MR).

c) Representative fluorescence staining for phagocytosis of green fluorescent latex beads. Cell cytoplasm stained using actin red.

(b-c) Scale bars represents $5 \mu \mathrm{m}$. Images acquired at $20 \mathrm{x}$ magnification.

d) Representative FACS histogram and quantification of BAL AM or exAM (2 months) for phagocytosis of pHRhodo Red Zymosan bioparticle conjugates at $4^{\circ} \mathrm{C}$ and $37^{\circ} \mathrm{C}$. Data shown are representative of 2 independent experiments.

e) Flow cytometric comparison of cell-surface markers F4/80, CD64, CD11c, SiglecF and $\mathrm{CD} 11 \mathrm{~b}$ expression on BAL AM and exAM. Colored lines indicate specific staining, grey areas indicate staining with isotype control antibody. Data shown are pooled from two independent experiments and representative of four independent experiments.

f) Representative fluorescence staining of BAL AM or exAM (2 months) for E.coli LPS (O55:B5) induced ROS production indicated by CM-H2 DCFDA staining (green).

g) RNA-seq gene expression of Core AM UP signature genes 3 in AM or exAM compared to $A M$ and peritoneal macrophages (PM) from Immgen consortium ${ }^{46}$ (GSE122108)

h) Heatmap of AM-specific transcription factor gene expression on AM or exAM compared to PM and AM from Immgen consortium $\underline{46}$ (GSE122108).

Figure 3. The exAM transcriptome shows adaptations to the culture environment

a) Volcano plot showing differential gene expression (DEGs, <0.05 FDR, $|F C|>2$ ) between exAM and in vivo AM (Host AM, Fig.4A).

b) Gene set enrichment analysis (GSEA) enrichment plot for GO biological process (GO: BP) positive regulation of mitotic cell cycle gene set (GO:0045931) in AM vs exAM. Corresponding leading-edge gene expression heatmap shown for AM vs exAM. 
c) Gene set enrichment analysis (GSEA) for Reactome: Signaling by TGF-b family members gene set (R-HAS-9006936) in AM vs exAM. Corresponding leading-edge gene expression heatmap shown for AM vs exAM.

d) Differentially expressed genes (DEGs) of AM or exAM in core AM signature genes 3 in ( $|F C|>4 ;<0.05$ FDR). Downregulated genes enriched for GO:BP lipid metabolic processes (GO:0006629, P-value 2.77E-8) marked by asterix*, AM core genes involved in lipid metabolism ${ }^{7}$ (in bold), potential niche interaction genes in blue.

e) K-means clustering $(K=3)$ heatmap showing total genes in AM vs exAM.

f) Gene ontology analysis on $\mathrm{K}$ means clusters $\mathrm{Cl}$ and $\mathrm{Cll}$ using GO:BP, KEGG, Reactome and Hallmark databases. Selection of representative, meaningful top hits.

g) Heatmaps of gene expression of selected representative key genes on recurring, complementary GO terms from analysis shown in Figure3F.

\section{Figure 4. Transplanted exAM restores full transcriptional AM identity in vivo}

a) Experimental setup for intra-tracheal (i.t) transplantation of freshly isolated BAL AM or 2 months expanded exAM (wild type, WT CD45.2) into lungs of WT CD45.1 host mice. Lungs analyzed 4 months post transplantation containing transplanted freshly isolated AM from BAL (tAM) or transplanted ex vivo amplified exAM (texAM) and resident host $A M$.

b) Contribution of CD45.2+ tAMs or texAMs and CD45.1 host AM to the total CD11c+SiglecF+ AM population, showing FACS example, individual values $(n=6)$. and median.

c) Surface marker expression of CD64, CD11c, SiglecF, CD11b and MHCII on host AM, tAM, and texAM. Data are representative of three independent experiments.

d) Heatmap of Spearman's correlation matrix with 2 biological replicates.

e) 3-dimentional principle component analysis (PCA) showing exAM (orange), texAM (red) and host AM (blue) compared to various tissue macrophage subsets 4 .

f) Overlaid volcano plot showing DEGs in tAM vs texAM in blue compared to exAM versus texAM in red (top), and texAM versu host $A M$ in blue compare to exAM versus host $A M$ in red (bottom) ( $|F C|>2,<0.05$ FDR).

g) Venn diagram showing overlap of DEGs up and down for exAM or tAM compared with texAM.

h) Venn diagrams showing total DEGs on pairwise comparisons on all in vivo AMs.

i) Heatmap showing core AM UP and DOWN signature gene expression-3 from our data compared to published tissue macrophage subsets 4 . Boxed outlines indicate restoration in vivo of gene expression of several Core AM UP genes shown to be altered in culture (Fig. 3D).

Figure 5. Epigenetic changes of expanded alveolar macrophages in culture are fully restored in vivo

a) Scatter plot analysis of ATAC-seq peaks differentially open or closed. Total peaks were pooled $(48,100)$ from three independent experiments with 2-3 biological replicates. Each point represents the average signal for each comparison.

b) Gene ontology analysis using GREAT tool $\underline{47}$ on differential open chromatin regions $(O C R)$ in exAM vs host AM. 
c) Examples of integrative genomics viewer (IGV) tracks for key genes linked to GO terms shown in Figure 5B and Figure 3D,F,G.

d) Venn diagram showing total differential peaks for exAM or texAM compared with Host AM.

e) Examples of integrative genomics viewer (IGV) tracks showing open chromatin regions (OCR) for AM specific transcription factors (TF) and cell surface markers and housekeeping genes (HKG) in our samples alongside various cell populations available through the Immgen consortium ${ }^{45}$. ATAC-seq signal pile-up traces were generated by pooling three independent experiment with 2-3 biological replicates each. Peak signals were group auto scaled based on data source.

Figure 6. Expanded alveolar macrophages show functional long-term contribution to alveolar macrophages in vivo

a) Schematic of intranasal transplant of exAM obtained from CD45.1 mice into empty niche of Csf2rb-/- CD45.2 neonates at post-natal day 1 and subsequent analysis at 3 months weeks or 8 months post transplantation.

b) Flow cytometric analysis of exAM contribution to Csf2rb-/- lungs at 8 months post exAM transplantation. FACS plots showing CD45.1 contribution of engrafted texAM (top panel) and SiglecF and CD11c AM surface markers (bottom panel).

c) Representative brightfield and Kwik-diff stained images of BAL at 8 months transplantation. WT (CD45.1) and Csf2rb-/- (CD45.2) BAL as controls.

d) Flow cytometric analysis of reduction of debris in BAL of Csf2rb-/- (CD45.2) mice post exAM transplantation. FACS plots showing debris clearance in exAM transplanted mice (top panel) and the \% debris (bottom panel) quantified in Figure $6 \mathrm{H}$.

e) Quantification of CD45.1+ exAM engraftment shown in Figure 6B and Fig.S7.

f) \%SiglecF+ CD11c+ AM in BAL corresponding to Figure 6B and Fig.S7.

g) Cell numbers counted in BAL corresponding to Fig 6C and Fig.S7.

h) Percent Debris and dead cells corresponding to Figure 6D and Fig.S7. Data in $(\mathrm{E}-\mathrm{H})$ shown as Mean $\pm \mathrm{SEM}$.

Figure 7. Schematic of epigenetic changes of exAM in culture and restoration in vivo

a) The scheme shows symbolic representation of AM identity OCR maintained across conditions, as well as culture adaptation OCR gained or natural environment induced OCR lost in culture and restored in the natural niche in vivo. 
1. Davies, L.C., Jenkins, S.J., Allen, J.E. \& Taylor, P.R. Tissue-resident macrophages. Nat Immunol 14, 986-995 (2013).

845

2. Bleriot, C., Chakarov, S. \& Ginhoux, F. Determinants of Resident Tissue Macrophage Identity and Function. Immunity 52, 957-970 (2020).

3. Gautier, E.L. et al. Gene-expression profiles and transcriptional regulatory pathways that underlie the identity and diversity of mouse tissue macrophages. Nat Immunol 13, 1118-1128 (2012).

852

5. Guilliams, M. \& Svedberg, F.R. Does tissue imprinting restrict macrophage plasticity?

6. Sieweke, M.H. Waddington's valleys and Captain Cook's islands. Cell Stem Cell 16, 7-8 (2015).

7. van de Laar, L. et al. Yolk Sac Macrophages, Fetal Liver, and Adult Monocytes Can

8. McQuattie-Pimentel, A.C. et al. The lung microenvironment shapes a dysfunctional response of alveolar macrophages in aging. J Clin Invest 131 (2021).

9. Gosselin, D. et al. Environment drives selection and function of enhancers controlling tissue-specific macrophage identities. Cell 159, 1327-1340 (2014).

10. Gosselin, D. et al. An environment-dependent transcriptional network specifies human microglia identity. Science 356 (2017).

11. Svedberg, F.R. et al. The lung environment controls alveolar macrophage metabolism and responsiveness in type 2 inflammation. Nat Immunol 20, 571-580 (2019).

12. Bohlen, C.J. et al. Diverse Requirements for Microglial Survival, Specification, and Function Revealed by Defined-Medium Cultures. Neuron 94, 759-773 e758 (2017).

13. Helft, J. et al. GM-CSF Mouse Bone Marrow Cultures Comprise a Heterogeneous Population of $\mathrm{CD} 11 \mathrm{C}(+) \mathrm{MHCII}(+)$ Macrophages and Dendritic Cells. Immunity 42, 11971211 (2015). elicited peritoneal macrophage cultures skews the functional readouts of in vitro assays. J Leukoc Biol 92, 325-331 (2012). 
15. Lim, W.A. \& June, C.H. The Principles of Engineering Immune Cells to Treat Cancer. Cell 168, 724-740 (2017).

891

16. Dolgin, E. Cancer-eating immune cells kitted out with CARs. Nat Biotechnol 38, 509-

17. Aziz, A., Soucie, E., Sarrazin, S. \& Sieweke, M.H. MafB/c-Maf deficiency enables selfrenewal of differentiated functional macrophages. Science 326, 867-871 (2009).

897

18. Soucie, E.L. et al. Lineage-specific enhancers activate self-renewal genes in 900

19. Busch, C.J., Favret, J., Geirsdottir, L., Molawi, K. \& Sieweke, M.H. Isolation and Longterm Cultivation of Mouse Alveolar Macrophages. Bio Protoc 9 (2019).

20. Schneider, C. et al. Induction of the nuclear receptor PPAR-gamma by the cytokine GMCSF is critical for the differentiation of fetal monocytes into alveolar macrophages. Nat Immunol 15, 1026-1037 (2014).

21. Aegerter, H. et al. Influenza-induced monocyte-derived alveolar macrophages confer prolonged antibacterial protection. Nat Immunol 21, 145-157 (2020).

22. Rauschmeier, R. et al. Bhlhe40 and Bhlhe41 transcription factors regulate alveolar macrophage self-renewal and identity. EMBO J 38, e101233 (2019).

23. Angelidis, l. et al. An atlas of the aging lung mapped by single cell transcriptomics and deep tissue proteomics. Nat Commun 10, 963 (2019).

24. Yu, X. et al. The Cytokine TGF-beta Promotes the Development and Homeostasis of Alveolar Macrophages. Immunity 47, 903-912 e904 (2017).

25. Misharin, A.V. et al. Monocyte-derived alveolar macrophages drive lung fibrosis and persist in the lung over the life span. J Exp Med 214, 2387-2404 (2017).

26. Machiels, B. et al. A gammaherpesvirus provides protection against allergic asthma by inducing the replacement of resident alveolar macrophages with regulatory monocytes. Nat Immunol 18, 1310-1320 (2017).

27. de Laval, B. et al. C/EBPbeta-Dependent Epigenetic Memory Induces Trained Immunity in Hematopoietic Stem Cells. Cell Stem Cell 26, 793 (2020).

28. Happle, C. et al. Pulmonary transplantation of macrophage progenitors as effective and long-lasting therapy for hereditary pulmonary alveolar proteinosis. Sci Trans/ Med 6, 250 ra113 (2014).

29. Suzuki, T. et al. Pulmonary macrophage transplantation therapy. Nature (2014). 
935

936

937

938

939

940

941

942

943

944

945

946

947

948

949

950

951

952

953

954

955

956

957

958

959

960

961

962

963

964

965

966

967

968

969

970

971

972

973

974

975

976

977

978

979

980

30. Guilliams, M. et al. Alveolar macrophages develop from fetal monocytes that differentiate into long-lived cells in the first week of life via GM-CSF. J Exp Med 210, 1977-1992 (2013).

31. Fuchs, E. The impact of cell culture on stem cell research. Cell Stem Cell 10, 640-641 (2012).

32. Heinz, S. et al. Simple combinations of lineage-determining transcription factors prime cis-regulatory elements required for macrophage and B cell identities. Mol Cell 38, 576589 (2010).

33. Heinz, S., Romanoski, C.E., Benner, C. \& Glass, C.K. The selection and function of cell type-specific enhancers. Nat Rev Mol Cell Biol 16, 144-154 (2015).

34. Glass, C.K. \& Natoli, G. Molecular control of activation and priming in macrophages. Nat Immunol 17, 26-33 (2016).

35. Xue, J. et al. Transcriptome-based network analysis reveals a spectrum model of human macrophage activation. Immunity 40, 274-288 (2014).

36. Yao, Y. et al. Induction of Autonomous Memory Alveolar Macrophages Requires T Cell Help and Is Critical to Trained Immunity. Cell 175, 1634-1650 e1617 (2018).

37. Bekkering, S. et al. Metabolic Induction of Trained Immunity through the Mevalonate Pathway. Cell 172, 135-146 e139 (2018).

38. Saeed, S. et al. Epigenetic programming of monocyte-to-macrophage differentiation and trained innate immunity. Science 345, 1251086 (2014).

39. Cheng, S.C. et al. mTOR- and HIF-1alpha-mediated aerobic glycolysis as metabolic basis for trained immunity. Science 345, 1250684 (2014).

40. Imperatore, F. et al. SIRT1 regulates macrophage self-renewal. EMBO J 36, 2353-2372 (2017).

41. Green, H. Cultured cells for the treatment of disease. Sci Am 265, 96-102 (1991).

42. Merad, M. \& Martin, J.C. Pathological inflammation in patients with COVID-19: a key role for monocytes and macrophages. Nat Rev Immunol 20, 355-362 (2020).

43. Robb, L. et al. Hematopoietic and lung abnormalities in mice with a null mutation of the common beta subunit of the receptors for granulocyte-macrophage colonystimulating factor and interleukins 3 and 5. Proc Natl Acad Sci U S A 92, 9565-9569 (1995). 
981

982

983

984

985

986

987

988

989

990

991

992

993

44. Corces, M.R. et al. Lineage-specific and single-cell chromatin accessibility charts human hematopoiesis and leukemia evolution. Nat Genet 48, 1193-1203 (2016).

45. Yoshida, H. et al. The cis-Regulatory Atlas of the Mouse Immune System. Cell 176, 897912 e820 (2019).

46. Gal-Oz, S.T. et al. ImmGen report: sexual dimorphism in the immune system transcriptome. Nat Commun 10, 4295 (2019).

47. McLean, C.Y. et al. GREAT improves functional interpretation of cis-regulatory regions. Nat Biotechnol 28, 495-501 (2010). 
Figure 1
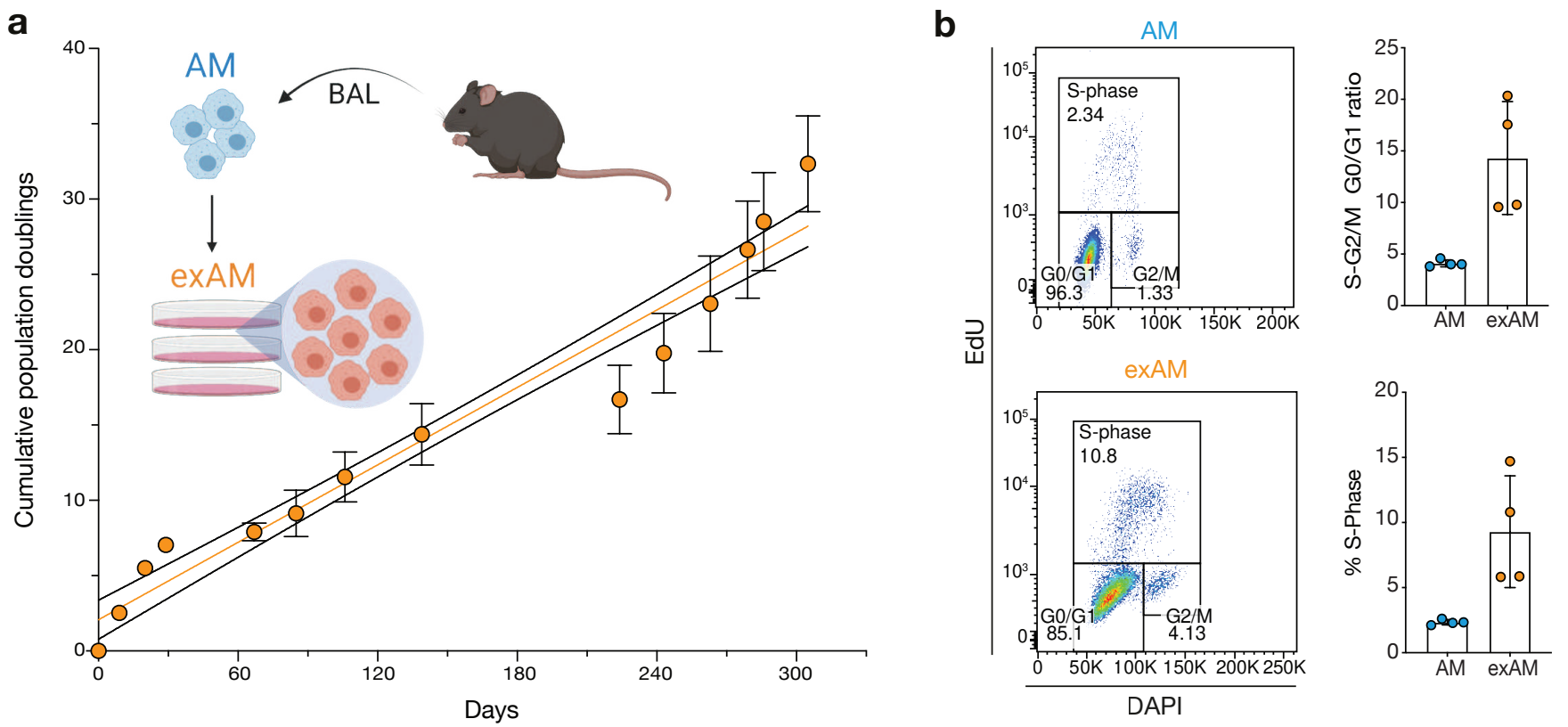
Figure 2

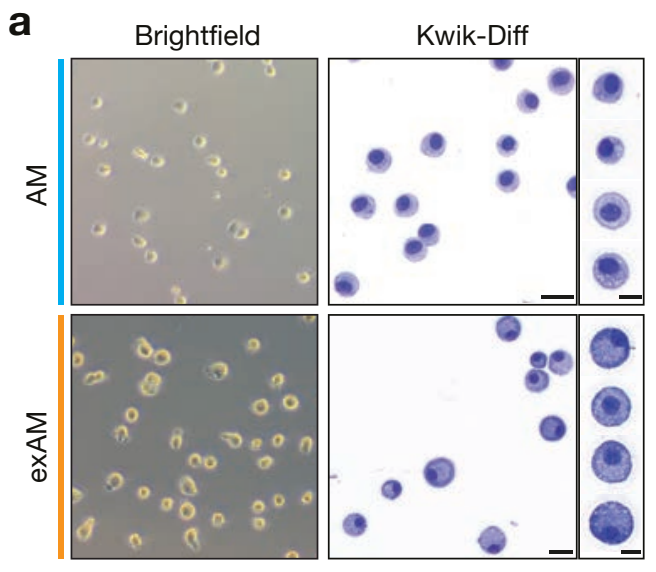

b

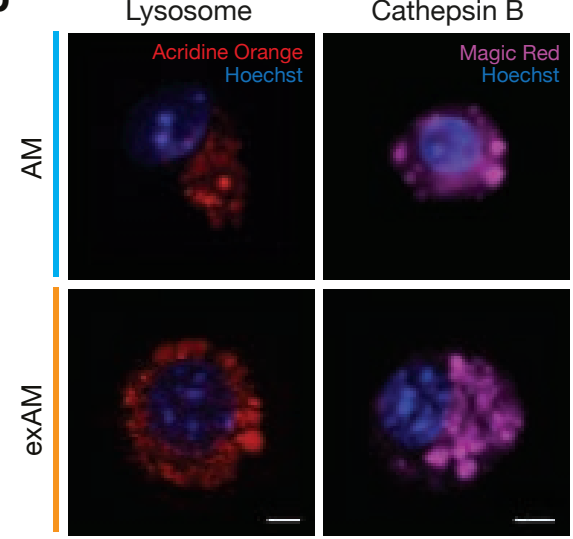

C Phagocytosis

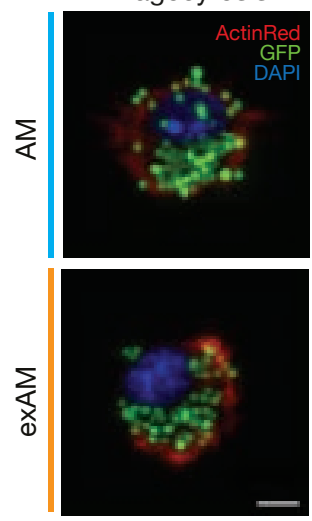

d
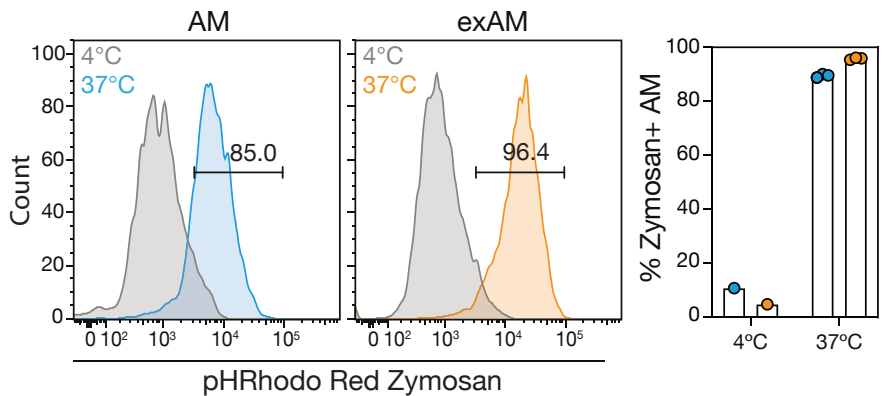

f

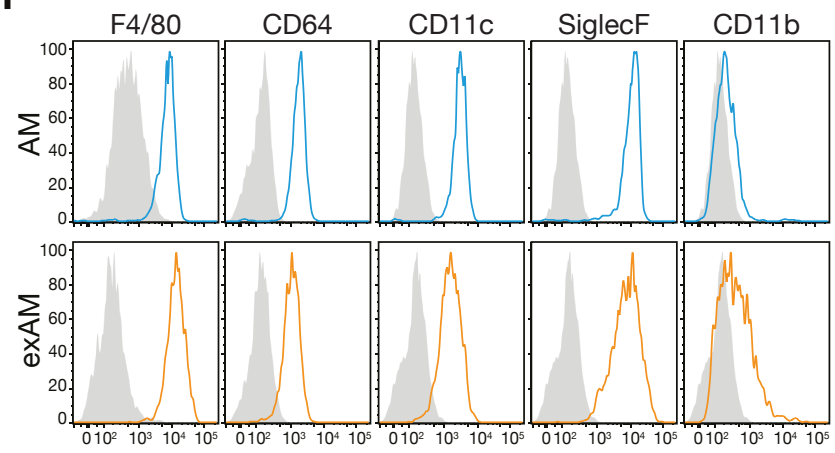

e

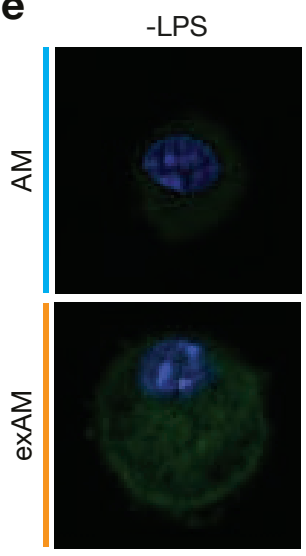

+ LPS

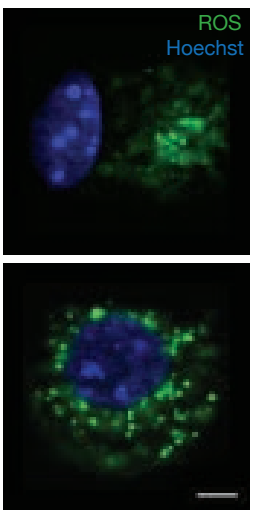

g

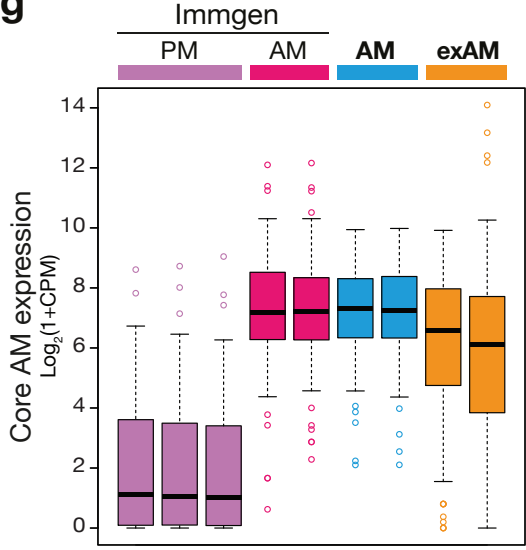

h

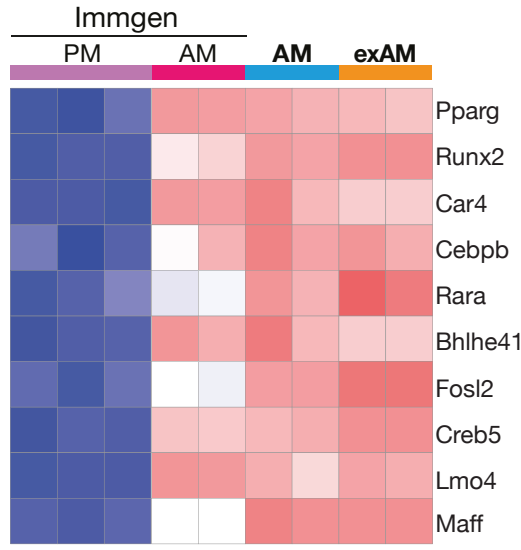




\section{Figure 3}

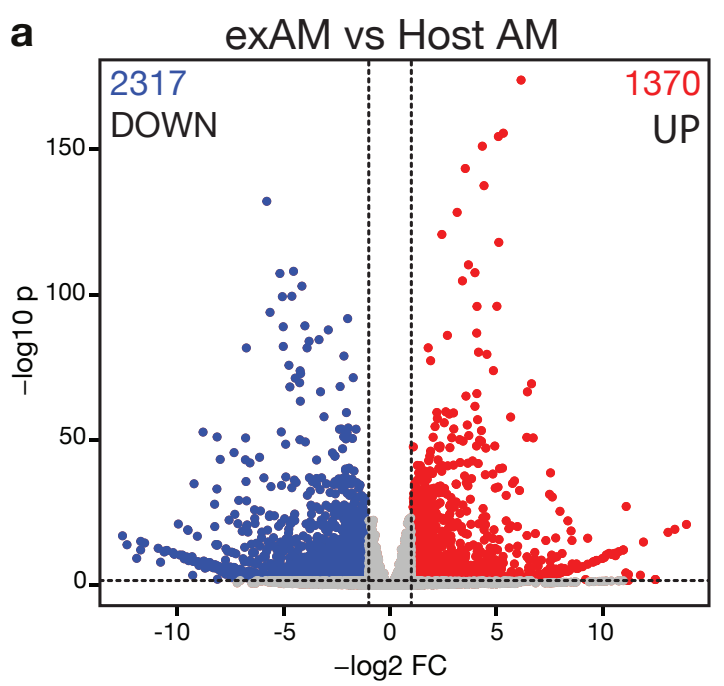

b Positive regulation of Positive regulation
mitotic cell cycle

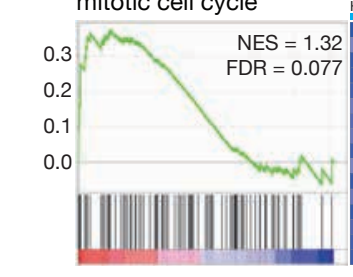

C Signaling by TGF-b

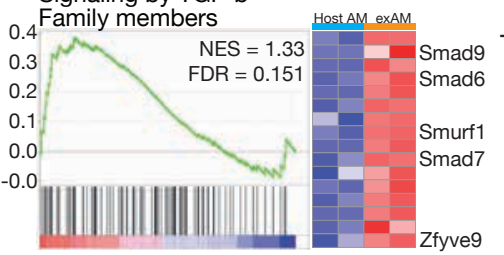

d

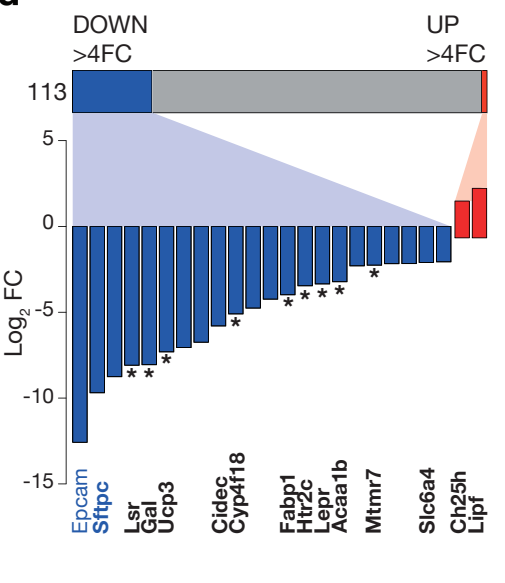

e
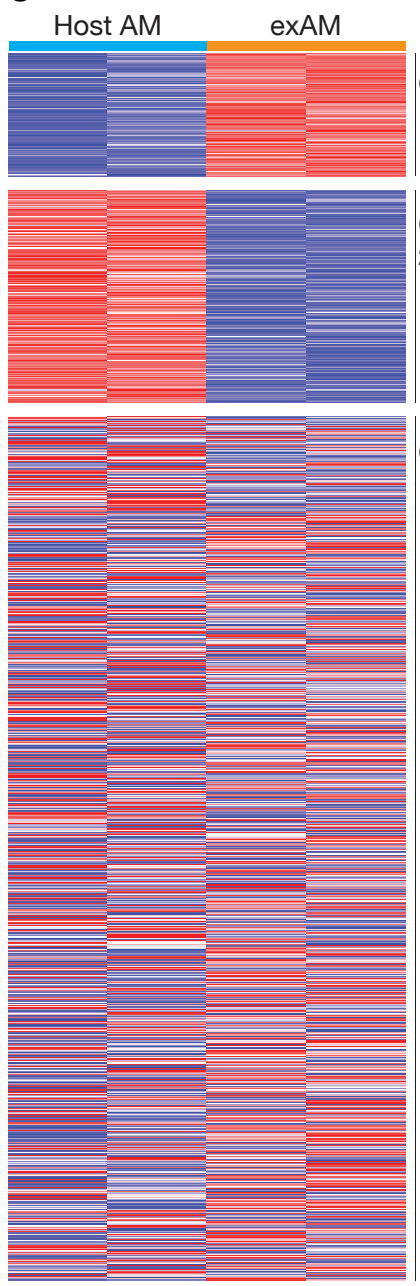

f

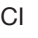

1384

CII

2374

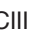

12646

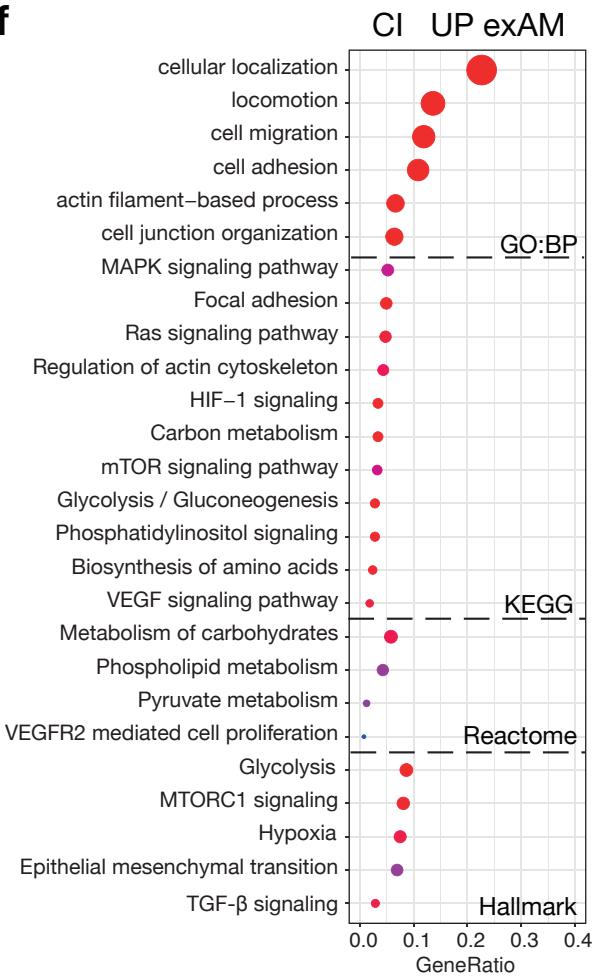

g

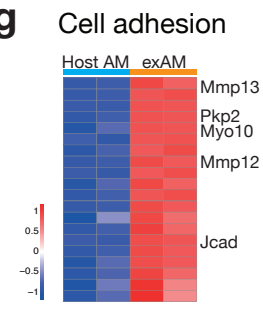

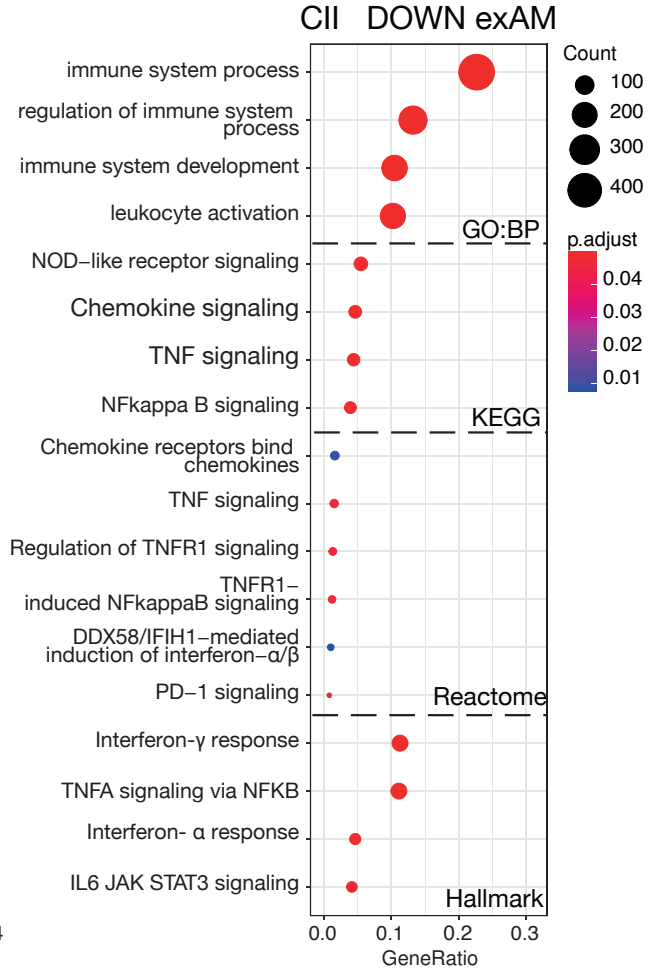

CII DOWN exAM

Glycolysis
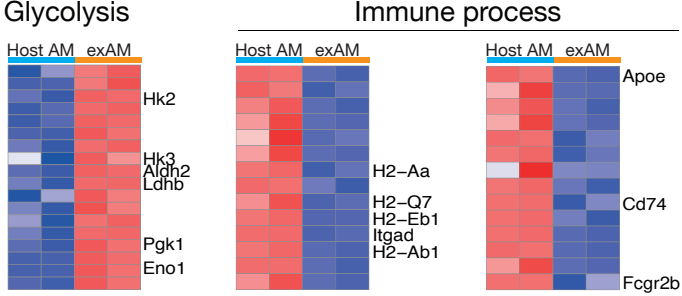
Figure 4

a
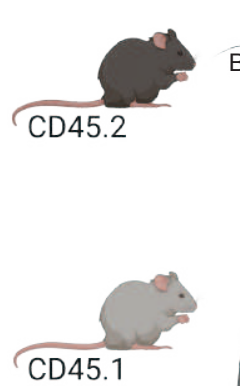

d

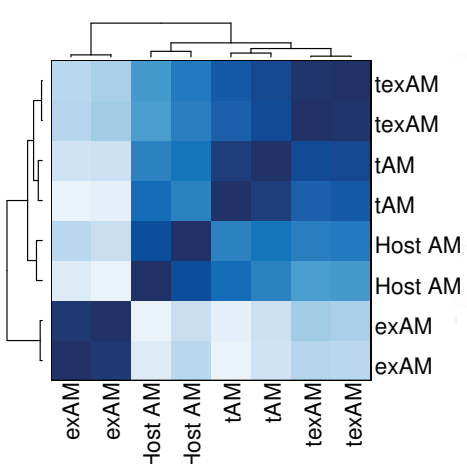

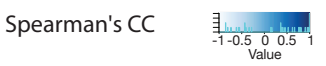
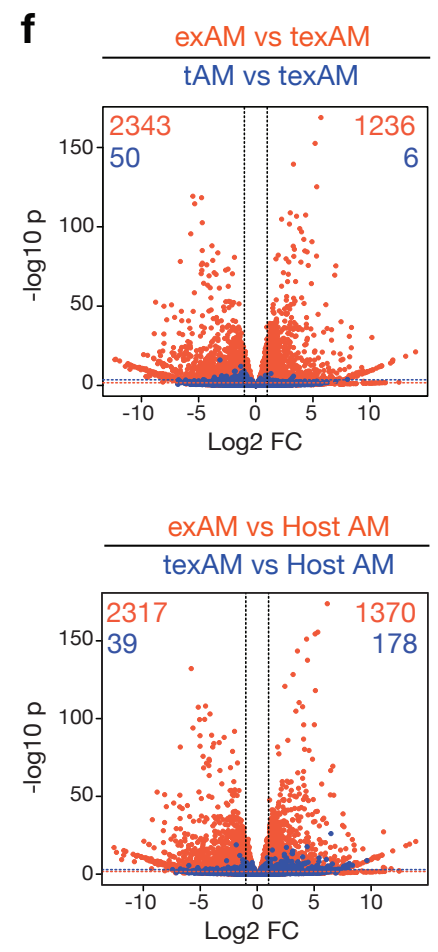

b
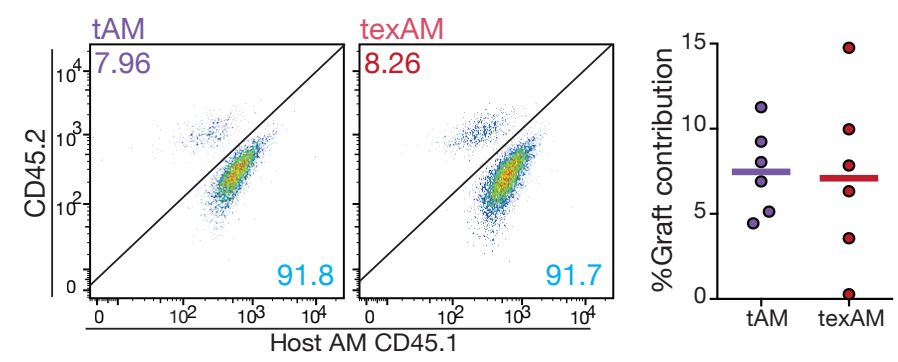

C

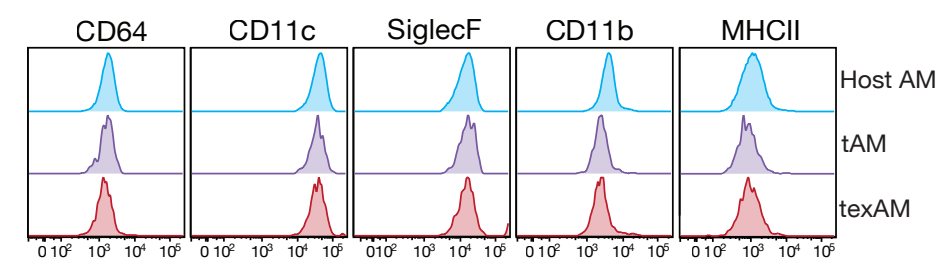

i

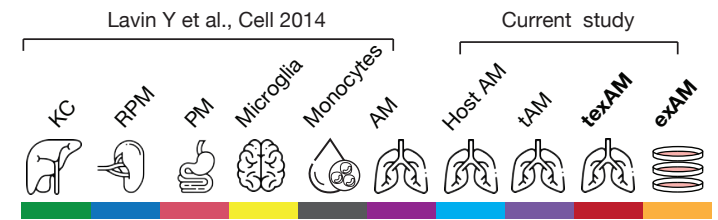

g exAM vs texAM

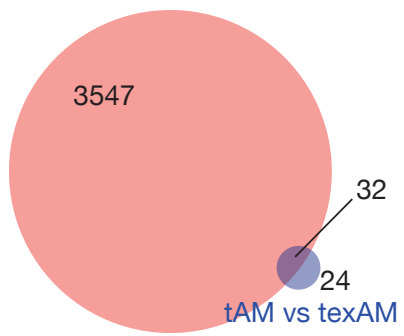

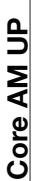

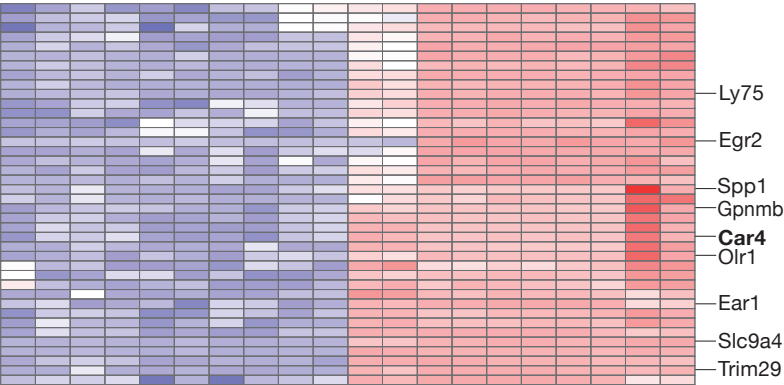

h

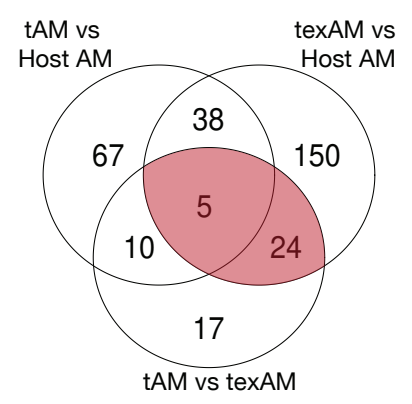

Egr2 Spp1 Car4 Ear1 Slc9a4
-Trim29
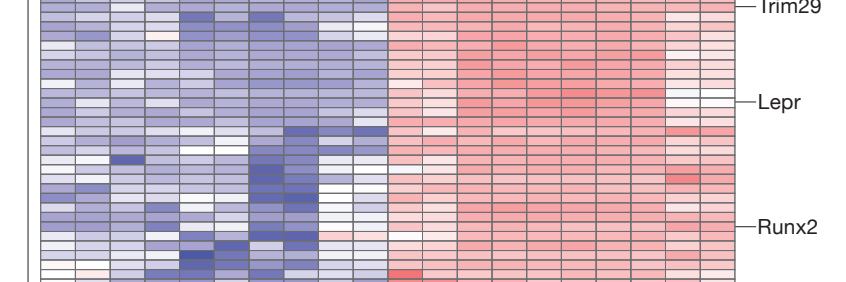
\begin{tabular}{|l|l|}
\hline \hline \hline$-C d 2$ \\
\hline \hline-
\end{tabular}

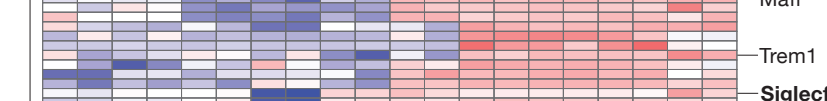

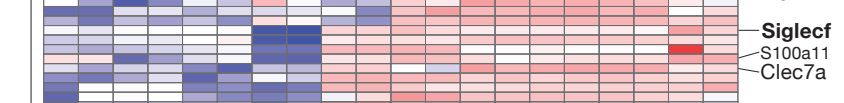
\begin{tabular}{|l|l|l|l|l|l|l|l}
\hline & Clec7a \\
\hline & & Naaa \\
\hline & & Ear2 \\
\hline
\end{tabular}

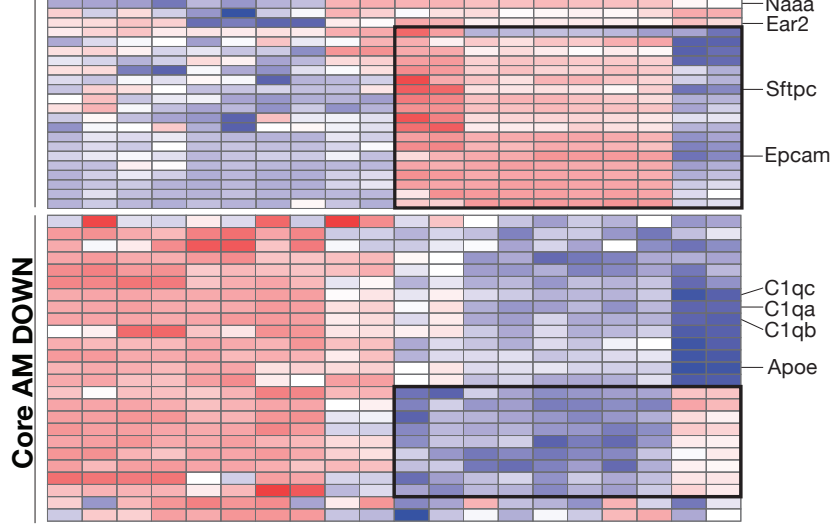




\section{Figure 5}

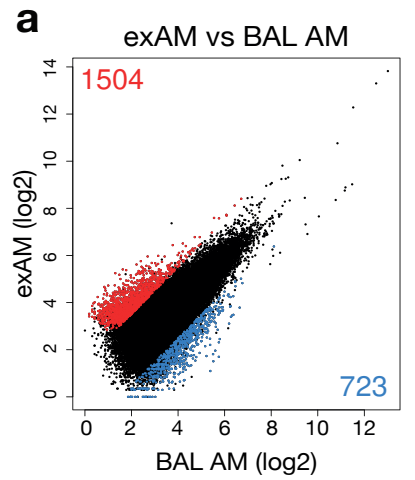

C

GO:BP

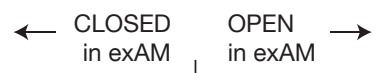

metabolic process

response to stress

regulation of mitotic cell cyclel

actin filament organization

regulation of cell shape

regulation of cell growth

cell junction organization

regulation of locomotion

regulation of cell migration

locomotion

cell motility,

regulation of actin
cytoskeleton organization ।

$$
\text { cell migration I }
$$
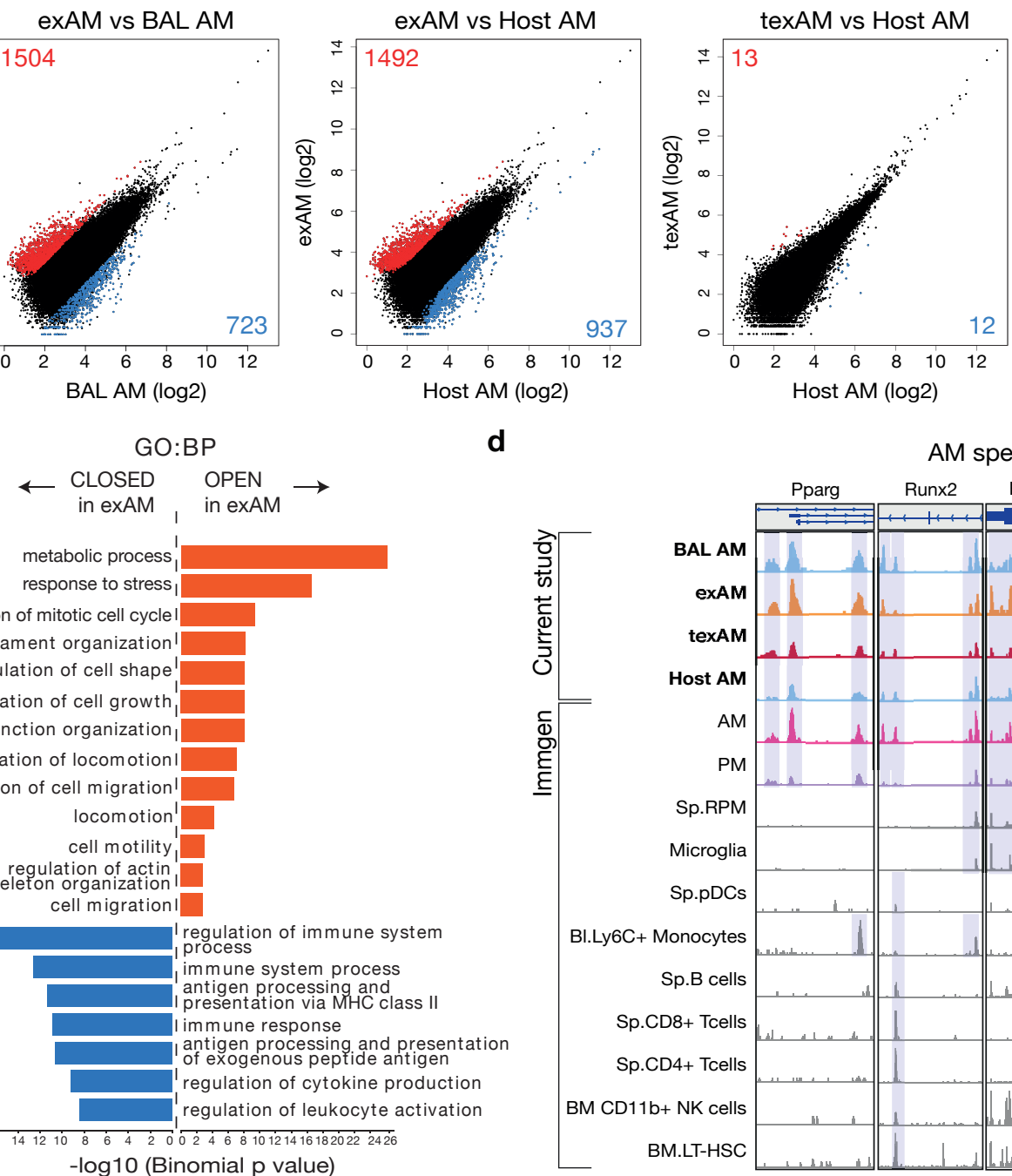

b

exAM vs Host AM

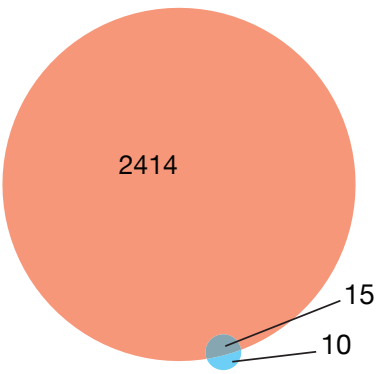

texAM vs Host AM

d

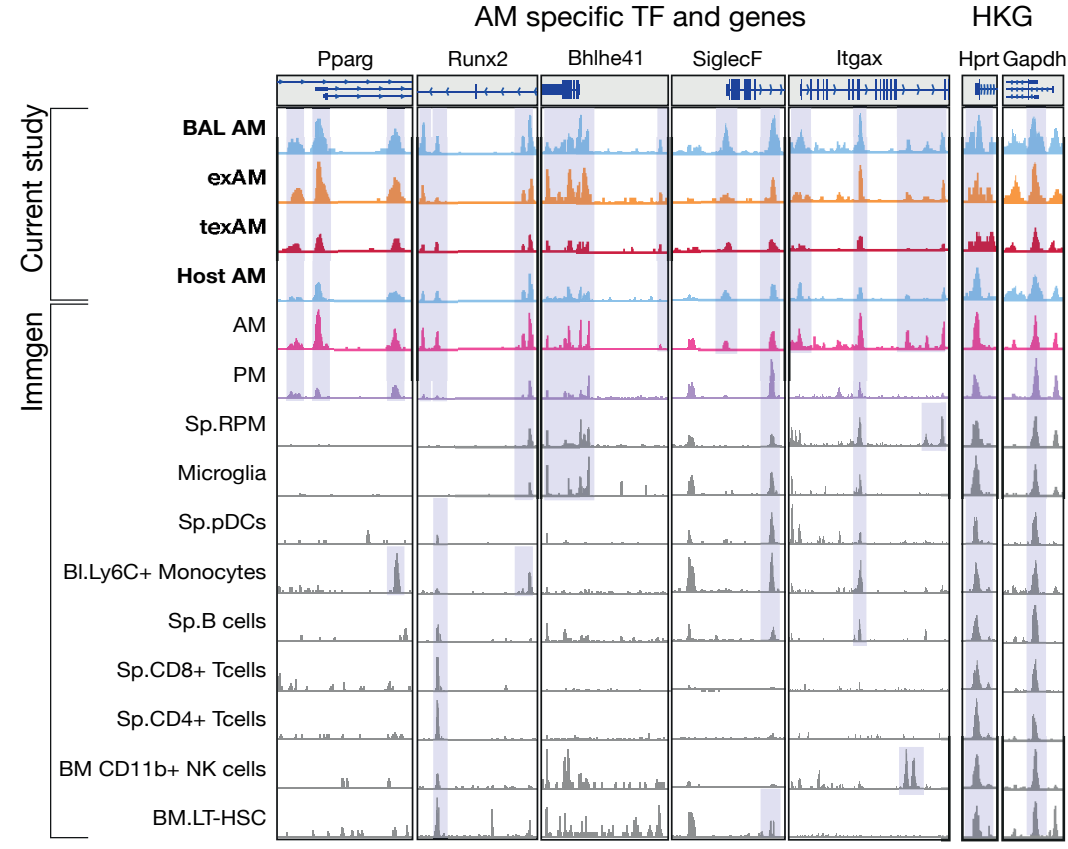

e Restoration of peaks in vivo

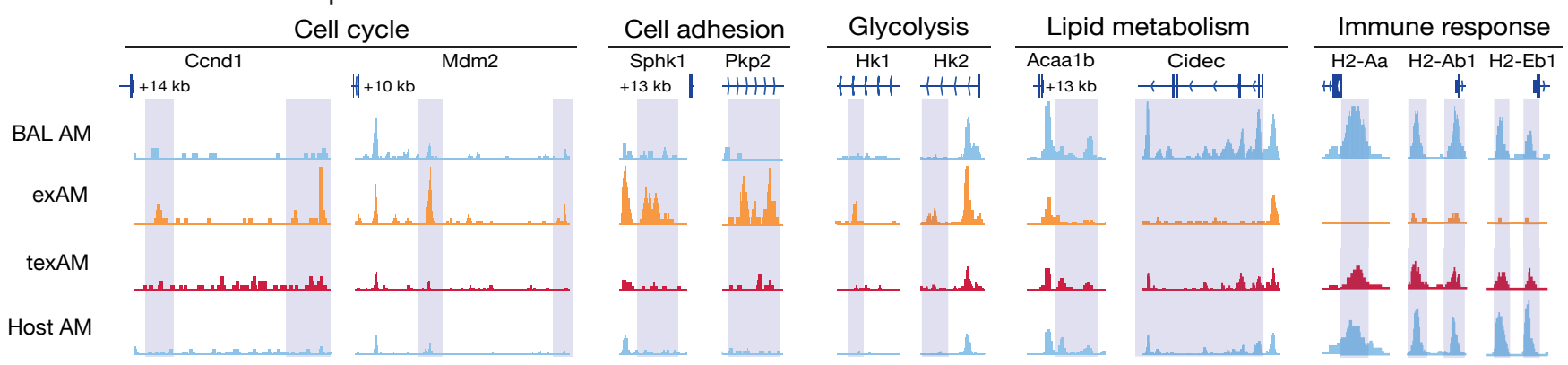


Figure 6

a
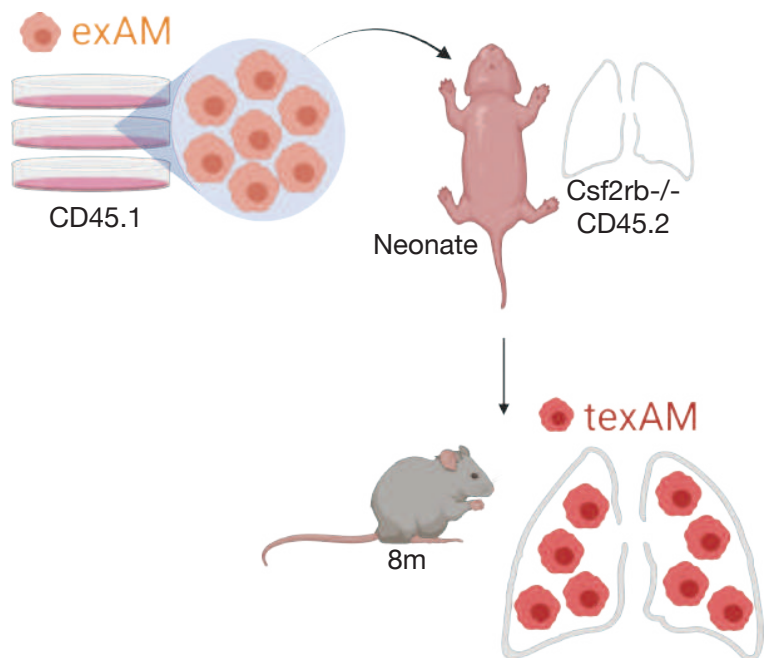

C
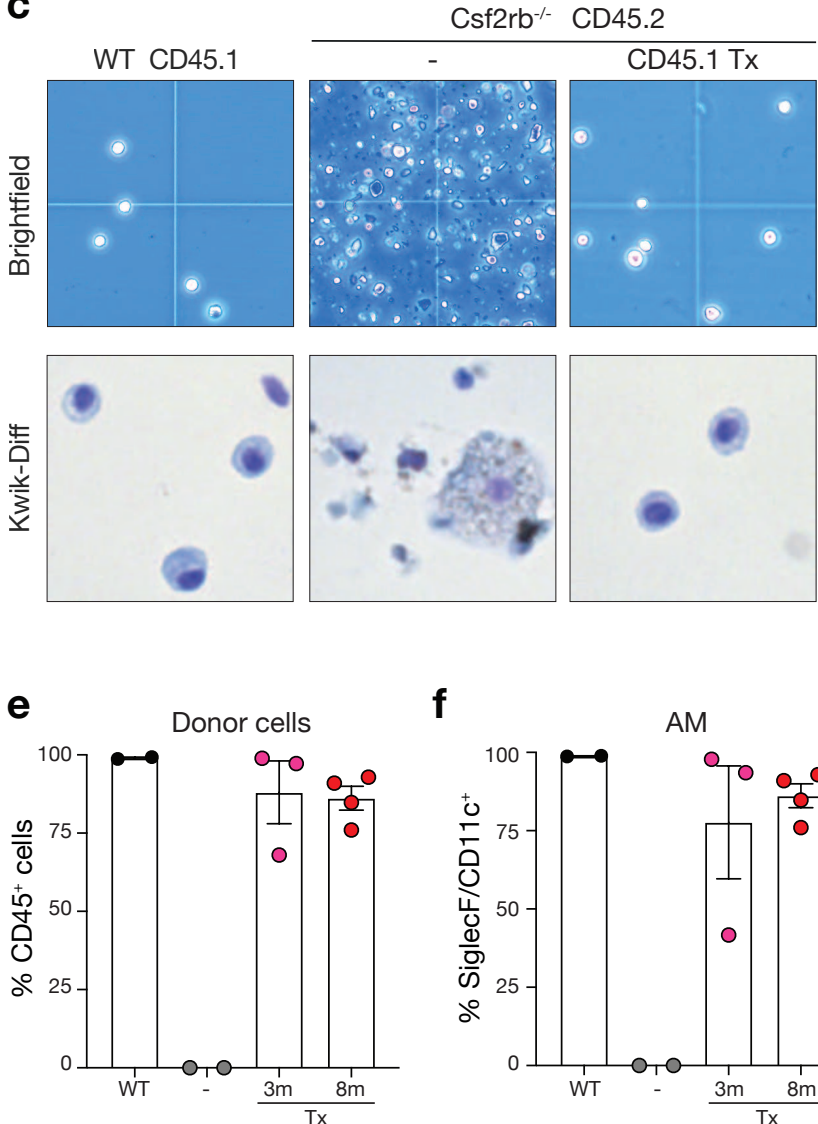

b
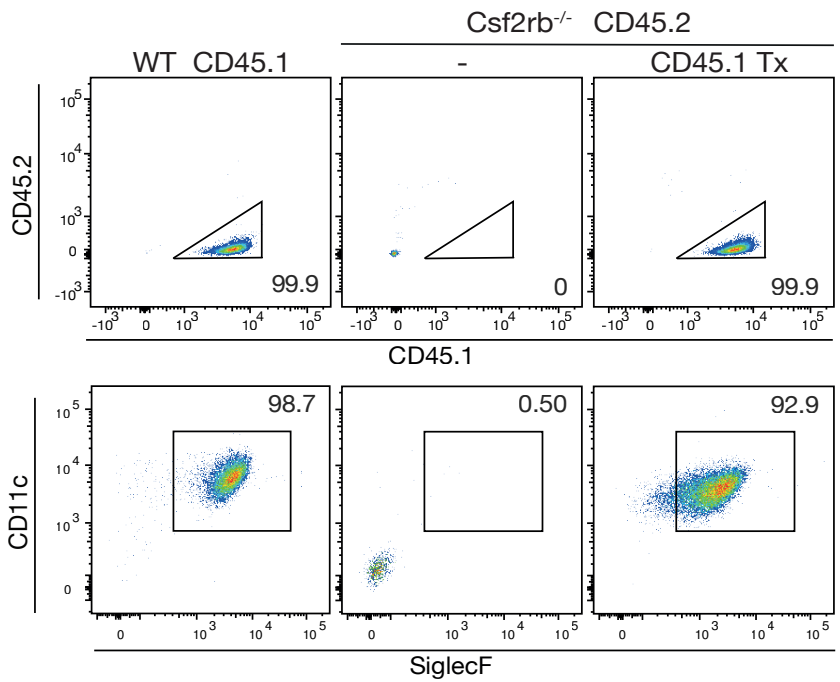

d
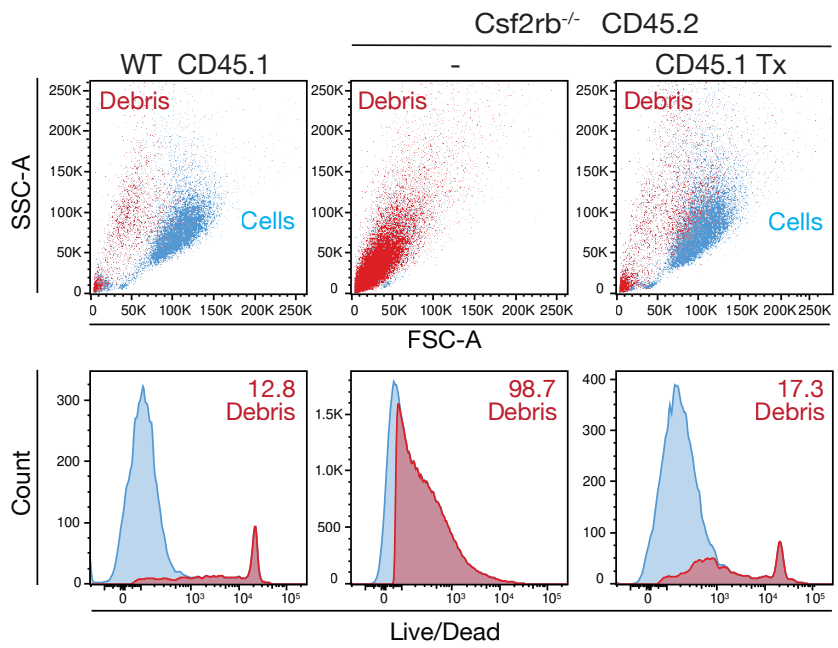

g

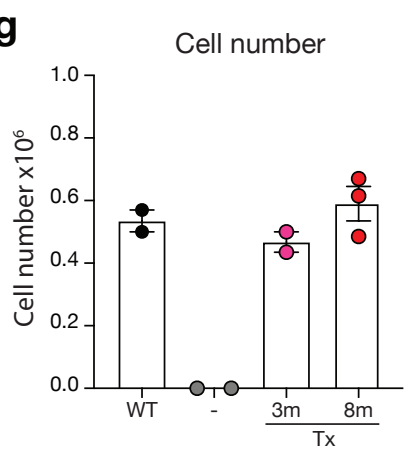

h

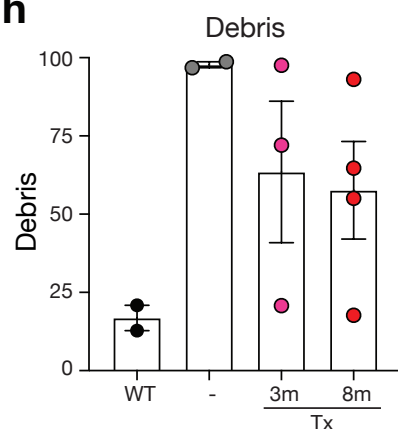




\section{Figure 7}

a

Alveolar Macrophages (AM)

Transplanted AM (tAM)

Transplanted expanded AM (texAM)
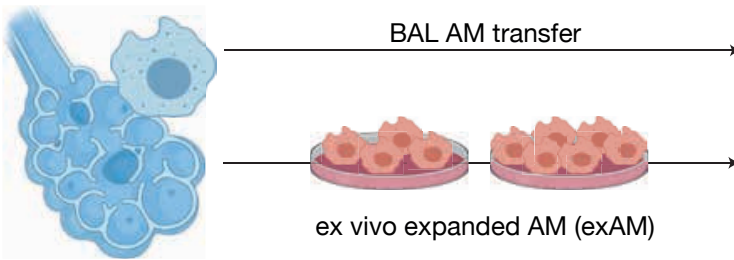

ex vivo expanded AM (exAM)

$$
\text { Host AM }
$$
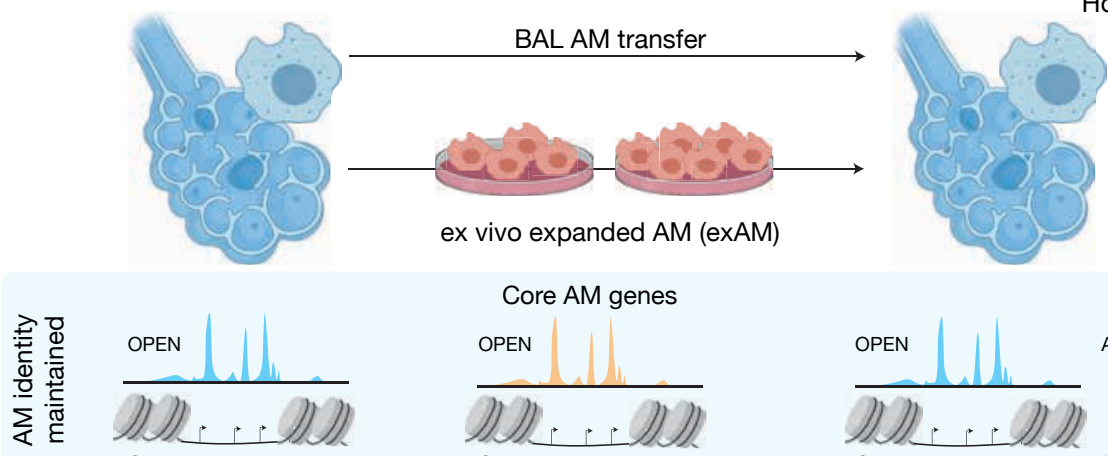

ON

$$
\text { ON }
$$

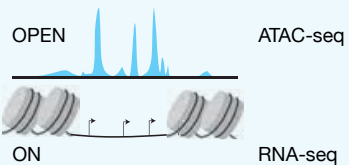

Cell Adhesion/Migration/Metabolism

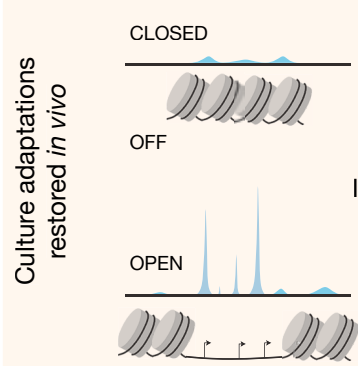

ON

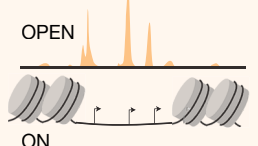

CLOSED

ATAC-seq

ON

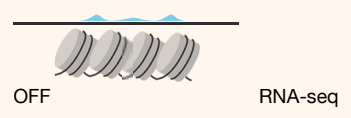

Immune system process/Lipid metabolism

CLOSED

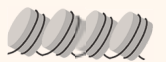

OFF

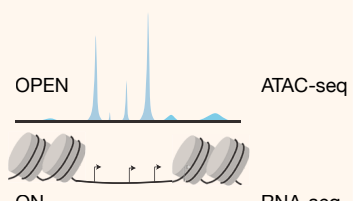

ON 


\section{Supplementary Files}

This is a list of supplementary files associated with this preprint. Click to download.

- 388460supp461600qwx2yhconvrt.pdf 